\title{
Kepler-21b: A Rocky Planet Around a V = 8.25 Mag Star
}

\section{Citation}

López-Morales, Mercedes, Raphaëlle D. Haywood, Jeffrey L. Coughlin, Li Zeng, Lars A. Buchhave, Helen A. C. Giles, Laura Affer, et al. 2016. “KEPLER-21b: A ROCKY PLANET AROUND AV= 8.25 Mag STAR." The Astronomical Journal 152 (6): 204. https:// doi.org/10.3847/0004-6256/152/6/204.

\section{Permanent link}

http://nrs.harvard.edu/urn-3:HUL.InstRepos:41412252

\section{Terms of Use}

This article was downloaded from Harvard University's DASH repository, and is made available under the terms and conditions applicable to Other Posted Material, as set forth at http:// nrs.harvard.edu/urn-3:HUL.InstRepos:dash.current.terms-of-use\#LAA

\section{Share Your Story}

The Harvard community has made this article openly available. Please share how this access benefits you. Submit a story.

Accessibility 
KEPLER-21b: A ROCKY PLANET AROUND A $V=8.25$ mag STAR*

\author{
Mercedes López-Morales ${ }^{1}$, Raphä̈lle D. Haywood ${ }^{1}$, Jeffrey L. Coughlin ${ }^{2}$, Li Zeng ${ }^{3}$, Lars A. Buchhave ${ }^{4}$, \\ Helen A. C. Giles ${ }^{5}$, Laura Affer ${ }^{6}$, Aldo S. Bonomo ${ }^{7}$, David Charbonneau ${ }^{1}$, Andrew Collier Cameron $^{8}$, \\ Rosario Consentino ${ }^{9}$, Courtney D. Dressing ${ }^{10,19}$, Xavier Dumusque ${ }^{5}$, Pedro Figueira ${ }^{11}$, Aldo F. M. Fiorenzano ${ }^{9}$, \\ Avet Harutyunyan ${ }^{9}$, John Asher Johnson ${ }^{1}$, David W. Latham ${ }^{1}$, Eric D. Lopez ${ }^{12}$, Christophe Lovis ${ }^{5}$, \\ Luca Malavolta $^{13,14}$, Michel Mayor ${ }^{5}$, Giusi Micela ${ }^{6}$, Emilio Molinari ${ }^{9,15}$, Annelies Mortier ${ }^{8}$, Fatemeh Motalebi $^{5}$, \\ Valerio Nascimbeni ${ }^{13}$, Francesco Pepe ${ }^{5}$, David F. Phillips ${ }^{1}$, Giampaolo Piotto ${ }^{13,14}$, Don Pollacco ${ }^{16}$, Didier Queloz $^{5,17}$, \\ Ken Rice ${ }^{12}$, Dimitar Sasselov ${ }^{1}$, Damien Segransan ${ }^{5}$, Alessandro Sozzetti ${ }^{7}$, Stephane Udry ${ }^{5}$, \\ ANDREW VANDERBURG ${ }^{1}$, AND Chris WATSON ${ }^{18}$ \\ ${ }^{1}$ Harvard-Smithsonian Center for Astrophysics, 60 Garden Street, Cambridge, MA 01238, USA; mlopez-morales@cfa.harvard.edu \\ ${ }^{2}$ SETI Institute, 189 Bernardo Avenue Suite 200, Mountain View, CA 94043, USA \\ ${ }^{3}$ Department of Earth and Planetary Sciences, Harvard University, 20 Oxford Street, Cambridge, MA 01238, USA \\ ${ }^{4}$ Centre for Star and Planet Formation, Natural History Museum of Denmark \& Niels Bohr Institute, University of Copenhagen, DK-1350 Copenhagen, Denmark \\ ${ }^{5}$ Observatoire Astronomique de 1'Université de Genéve, Chemin des Maillettes 51, Sauverny, CH-1290, Switzerland \\ ${ }^{6}$ INAF-Osservatorio Astronomico di Palermo, Piazza del Parlamento 1, I-90124 Palermo, Italy \\ ${ }^{7}$ INAF-Osservatorio Astrofisico di Torino, via Osservatorio 20, I-10025 Pino Torinese, Italy
${ }^{8}$ SUPA, School of Physics \& Astronomy, University of St. Andrews, North Haugh, St. Andrews Fife, KY16 9SS, UK \\ INAF-Fundación Galileo Galilei, Rambla José Ana Fernandez Pérez 7, E-38712 Breña Alta, Spain \\ ${ }^{10}$ Division of Geological and Planetary Sciences, California Institute of Technology, Pasadena, CA 91125, USA \\ ${ }^{11}$ Instituto de Astrofísica e Ciências do Espaço, Universidade do Porto, CAUP, Rua das Estrelas, PT4150-762 Porto, Portugal \\ ${ }^{12}$ SUPA, Institute for Astronomy, University of Edinburgh, Royal Observatory, Blackford Hill, Edinburgh, EH93HJ, UK \\ ${ }^{13}$ Dipartimento di Fisica e Astronomia "Galileo Galilei," Universita'di Padova, Vicolo dell'Osservatorio 3, I-35122 Padova, Italy \\ ${ }^{14}$ INAF-Osservatorio Astronomico di Padova, Vicolo dell'Osservatorio 5, I-35122 Padova, Italy \\ ${ }^{15}$ INAF-IASF Milano, via Bassini 15, I-20133, Milano, Italy \\ ${ }^{16}$ Department of Physics, University of Warwick, Gibbet Hill Road, Coventry CV4 7AL, UK \\ ${ }^{17}$ Cavendish Laboratory, J J Thomson Avenue, Cambridge CB3 OHE, UK \\ ${ }^{18}$ Astrophysics Research Centre, School of Mathematics and Physics, Queens University, Belfast, Belfast BT7 1NN, UK \\ Received 2016 August 15; revised 2016 September 14; accepted 2016 September 22; published 2016 December 2
}

\begin{abstract}
HD 179070, aka Kepler-21, is a $V=8.25$ F6IV star and the brightest exoplanet host discovered by Kepler. An early detailed analysis by Howell et al. of the first 13 months (Q0-Q5) of Kepler light curves revealed transits of a planetary companion, Kepler-21b, with a radius of about $1.60 \pm 0.04 R_{\oplus}$ and an orbital period of about 2.7857 days. However, they could not determine the mass of the planet from the initial radial velocity (RV) observations with Keck-HIRES, and were only able to impose a $2 \sigma$ upper limit of $10 M_{\oplus}$. Here, we present results from the analysis of 82 new RV observations of this system obtained with HARPS-N, together with the existing 14 HIRES data points. We detect the Doppler signal of Kepler-21b with a RV semiamplitude $K=2.00 \pm 0.65 \mathrm{~m} \mathrm{~s}^{-1}$, which corresponds to a planetary mass of $5.1 \pm 1.7 M_{\oplus}$. We also measure an improved radius for the planet of $1.639+0.019 /-0.015 R_{\oplus}$, in agreement with the radius reported by Howell et al. We conclude that Kepler- $21 \mathrm{~b}$, with a density of $6.4 \pm 2.1 \mathrm{~g} \mathrm{~cm}^{-3}$, belongs to the population of small, $\lesssim 6 M_{\oplus}$ planets with iron and magnesium silicate interiors, which have lost the majority of their envelope volatiles via stellar winds or gravitational escape. The RV analysis presented in this paper serves as an example of the type of analysis that will be necessary to confirm the masses of TESS small planet candidates.
\end{abstract}

Key words: planets and satellites: formation - planets and satellites: individual (Kepler-21b) -

stars: individual (HD 179070) - techniques: photometric - techniques: radial velocities - techniques: spectroscopic

Supporting material: machine-readable table

\section{INTRODUCTION}

Results from NASA's Kepler Satellite Mission have revealed an abundance of planets smaller than $2 R_{\oplus}$ with orbital periods less than 100 days (Howard et al. 2012; Dressing \& Charbonneau 2013; Fressin et al. 2013; Petigura et al. 2013a, 2013b; Foreman-Mackey et al. 2014; Dressing \& Charbonneau 2015; Silburt et al. 2015). Although only a few of

* Based on observations made with the Italian Telescope Nazionale Galileo (TNG) operated on the island of La Palma by the Fundación Galileo Galilei of the INAF (Istituto Nazionale di Astrofisica) at the Spanish Observatorio del Roque de los Muchachos of the Instituto de Astrofísica de Canarias.

19 NASA Sagan Fellow. those planets have measured masses, and therefore densities, those measurements have started to unveil an interesting picture. Below a radius of about $1.6 R_{\oplus}$ most planets are consistent with bare rocky compositions without any significant volatile envelopes (Rogers 2015). Moreover, when considering only planets with masses measured with precisions better than $20 \%$ via RVs, planets with masses smaller than about $6 M_{\oplus}$ appear to be rocky and have interiors composed mostly of iron and magnesium silicates in Earth-like abundances $(26 \% \mathrm{Fe}$, $74 \% \mathrm{MgSiO}_{3}$, on average, based on Zeng et al. 2016), while planets more massive than about $7 M_{\oplus}$ show a wider range of densities (Dressing et al. 2015; Gettel et al. 2016). Such dichotomy suggests the possible existence of mechanisms by 
which planets more massive than approximately $7 M_{\oplus}$ in orbits of only a few days, can retain significant volatile envelopes, while less massive planets lose all the material in their outer layers to a combination of the effect of stellar winds and atmospheric escape.

However, despite the rapid observational progress on the determinaiton of fundamental properties of low-mass planets, some basic questions about the origin of this short-period rocky planet population are still not understood. Almost all of the confirmed rocky planets are on highly irradiated orbits, where they are bombarded by large amounts of ionizing EUV and $\mathrm{X}$-ray radiation, which can drive a photoevaporative wind from the atmosphere of the planet and over a planet's lifetime can remove a significant amount of mass from planets with volatile envelopes (e.g., Owen \& Jackson 2012). Several recent studies have shown that Kepler's short-period super-Earths and subNeptunes have likely been significantly sculpted by photoevaporation (e.g., Lopez et al. 2012; Lopez \& Fortney 2013; Owen \& Wu 2013), or else by some other comparable process like atmospheric erosion by impacts (e.g., Inamdar \& Schlichting 2015; Schlichting et al. 2015). Thus, while it is possible that the short-period rocky planets simply formed with their current Earth-like compositions, their low masses and highly irradiated orbits mean that they could also be the remnant cores of volatile-rich hot Neptunes, which have lost their envelopes. Even considering all these scenarios, it is not clear why a transition between bare cores and planets with significant volatiles would occur at $1.6 R_{\oplus}$. For example, recent precise mass measurements of planets with masses between 3 and $8 \quad M_{\oplus}$ and periods up to 17 days, via transit timing variations, reveal a wide range of densities for planets with masses near 5-6 $M_{\oplus}$, analogous to the situation for more massive planets (Jontof-Hutter et al. 2016). The recently discovered Kepler-20b, with a mass of $9.7 M_{\oplus}$, radius $1.9 R_{\oplus}$, and a orbital period of 3.7 days appears to be a bare core (Buchhave et al. 2016).

With the current sample of small planets with precise mass measurements it is not possible to establish whether stellar irradiation is the cause of the bare core to volatile-rich envelopes transition. It is also not possible to discern whether the transition is abrupt or smooth (Rogers 2015). We, therefore, need a larger number of precise mass measurements, especially around the apparent $1.6 R_{\oplus}$ transition region.

In this paper, we report the mass measurement of Kepler$21 \mathrm{~b}$, a $5.1+/-1.7 M_{\oplus}$ super-Earth located at the apparent mass boundary between predominately volatile-poor superEarths and volatile-rich larger planets. Kepler-21b orbits the brightest exoplanet host star discovered by Kepler (HD $179070, V=8.25)$, which is also a slightly evolved F6IV star. An earlier study of this planet by Howell et al. (2012), based on the first six quarters of Kepler data (Q0-Q5), found a planet radius of $1.6 \pm 0.04 R_{\oplus}$, but could not determine the planetary mass, because of the effect of the stellar variability in the radial velocity (RV) measurements. Our mass measurement comes from new RV data collected with HARPS-N between 2014 and 2015, combined with the HIRES data from Howell et al. (2012) and fitted using Gaussian process regression (hereafter referred to as GP; see Rasmussen \& Williams 2006, for more details). In addition, we compute a new planetary radius from the complete Kepler Q0-Q17 light curves, detrended from stellar variability using new time series analysis techniques.
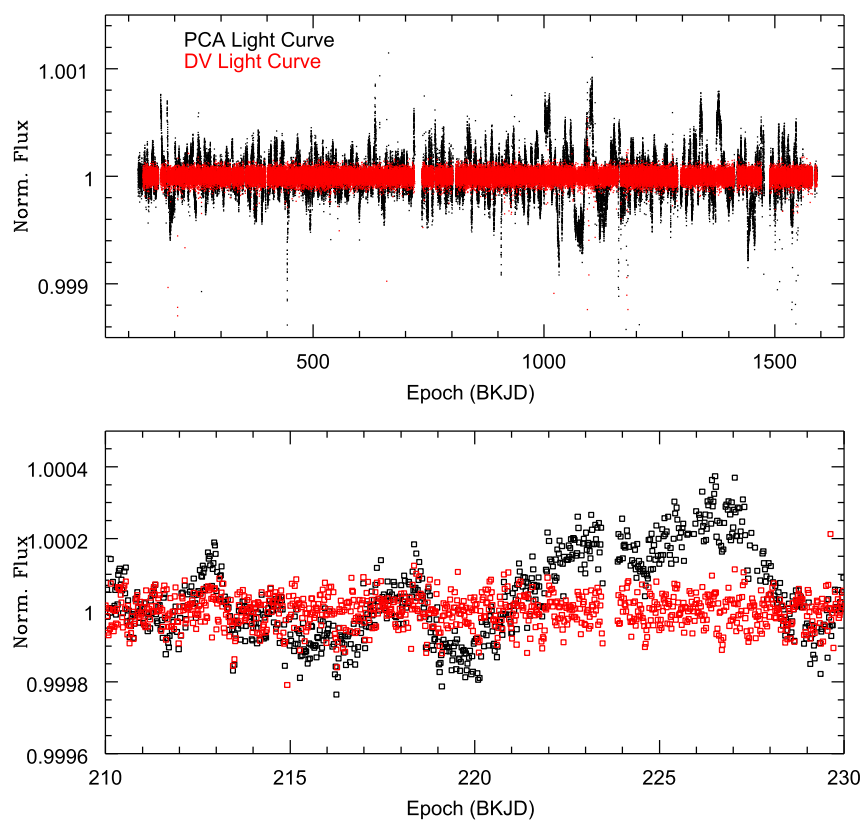

Figure 1. (Top) Full Q0-Q17 Kepler light curve analyzed using DV (red) and PCA (black) detrendings. (Bottom) A 20 day zoom-in of the light curves to illustrate the difference between the DV and the PCA detrendings, with the latter preserving the variability signal of the star. The times are given in Barycentric Kepler Julian Dates (BKJDs), i.e., BJD—2454833.0.

We describe the light curve and RV analyses in Section 2. In Sections 3 and 4, we describe the light curve and RV fits and their results. Finally, we discuss our findings and summarize our conclusions in Section 5.

\section{DATA}

\subsection{Kepler Photometry}

Kepler-21 was monitored with Kepler in 29.4 minute, longcadence mode between quarters Q0 and Q17, and in $58.9 \mathrm{~s}$, short cadence mode in quarters Q2 and Q5-Q17, covering a total time period of 1470.5 days (BJD 2454953.540-2456424.002). We analyzed the full Kepler data set using two different detrendings: Data validation (DV) and principal component analysis (PCA). The results of both analyses are shown in Figure 1.

The result of the DV analysis is the detrended flux time series available in the DV report summaries (Wu et al. 2010) as obtained from the NASA Exoplanet Archive's Q1-Q17 DR24 TCE table. ${ }^{19}$ For this detrending, as detailed in Jenkins et al. (2010), an optimal photometric aperture is used to sum up the flux from the central pixels of the pixel-stamp image and produce a time series light curve. The Pre-Search Data Conditioning (PDC) module then removes systematic trends, which are common to multiple stars on the detector. The resulting time series is then run through a harmonic filter, which identifies and removes sinusoidal trends in the data. Finally, a median detrender is used to remove any remaining photometric variations at durations larger than the transit duration and normalize the data (see Wu et al. 2010, for more details).

While the DV detrending produces a very clean light curve, any variations at timescales greater than the transit duration,

\footnotetext{
${ }^{19}$ http://exoplanetarchive.ipac.caltech.edu
} 

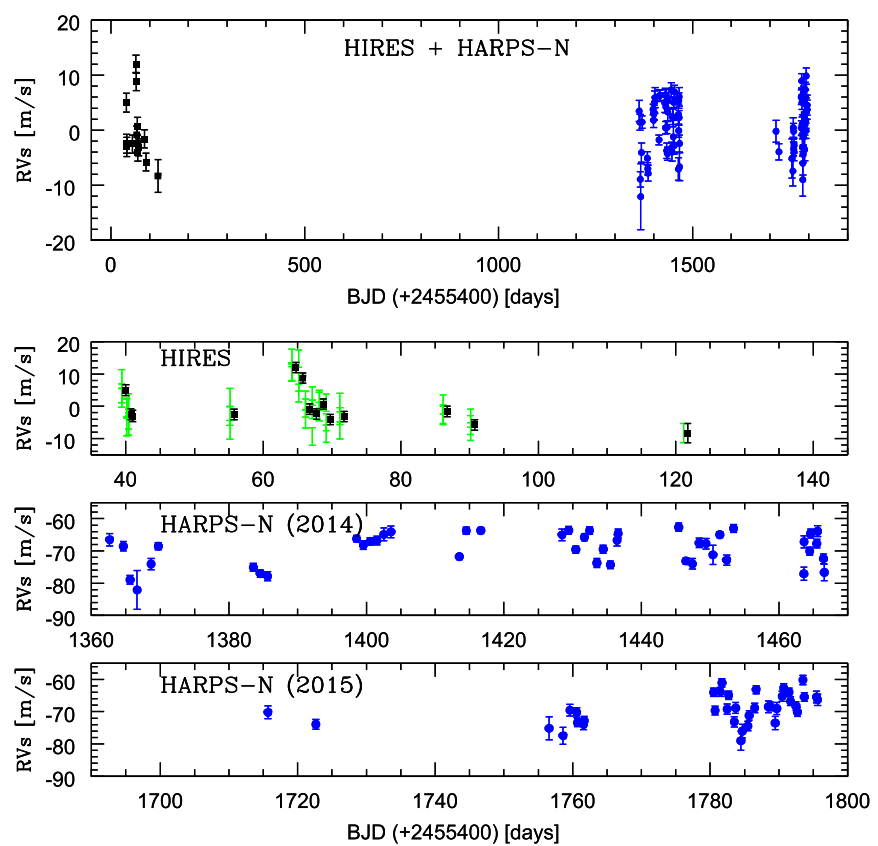

Figure 2. The top diagram shows the combined HIRES (black squares) and HARPS-N (blue circles) RV observations corrected from a systemic velocity for the star of $19,100 \mathrm{~m} \mathrm{~s}^{-1}$ and for offsets between the data sets. The bottom three diagrams show the HIRES data and the HARPS-N 2014 and 2015 data separately, plotted over the same timescale (110 days). The green lines in the HIRES plot show the individual 2-3 minute observations. The black squares show the weighted average of each set of three consecutive observations, as reported by Howell et al. (2012). The green lines have been shifted by -0.6 BJDs for clarity. The difference in the vertical scales between the HIRES and the HARPS-N plots shows the instrumental offset of $70 \mathrm{~m} \mathrm{~s}^{-1}$ between both data sets.

such as the planet's phase curve or stellar variations due to rotation or pulsation, are removed. This is due mainly to the harmonic filter and median detrender, which are selected to preserve features with timescales in the order of the transits. Detailed inspection of the light curves also shows that the PDC module significantly suppresses sinusoidal-like astrophysical signals at 10 days, and completely removes them by 20 days (Christiansen et al. 2013). Stellar rotation periods, which can be confused with an exoplanet's RV signal, are usually in that same 10-20 day period range (see e.g., McQuillan et al. 2014), so it is important to preserve the stellar signal. Therefore, we also employed a PCA detrending (Murtagh \& Heck 1987), similar to that described in Coughlin \& López-Morales (2012). ${ }^{20}$ For the PCA detrending, all available pixels in the pixel-stamp image are summed up to produce a time series light curve. A PCA is then run on the pixel-level time series data to obtain a series of basis vector components. These components correspond to the systematic trends belonging to the specific target being analyzed, which arise due to motion on the detector, as well as instrumental variation and cosmic ray impacts. These basis vectors are removed from the time series photometry, which is then normalized by simply dividing by the median flux level in each quarter. The advantage of this PCA detrending is that it preserves the intrinsic photometric signals introduced by both the star and the planet, while removing systematic trends from the spacecraft and detector.

\footnotetext{
${ }^{20}$ This tool is now publicly available as a task called keppca in the Kepler PyKE tools package (http://keplergo.arc.nasa.gov/PyKE.shtml).
}
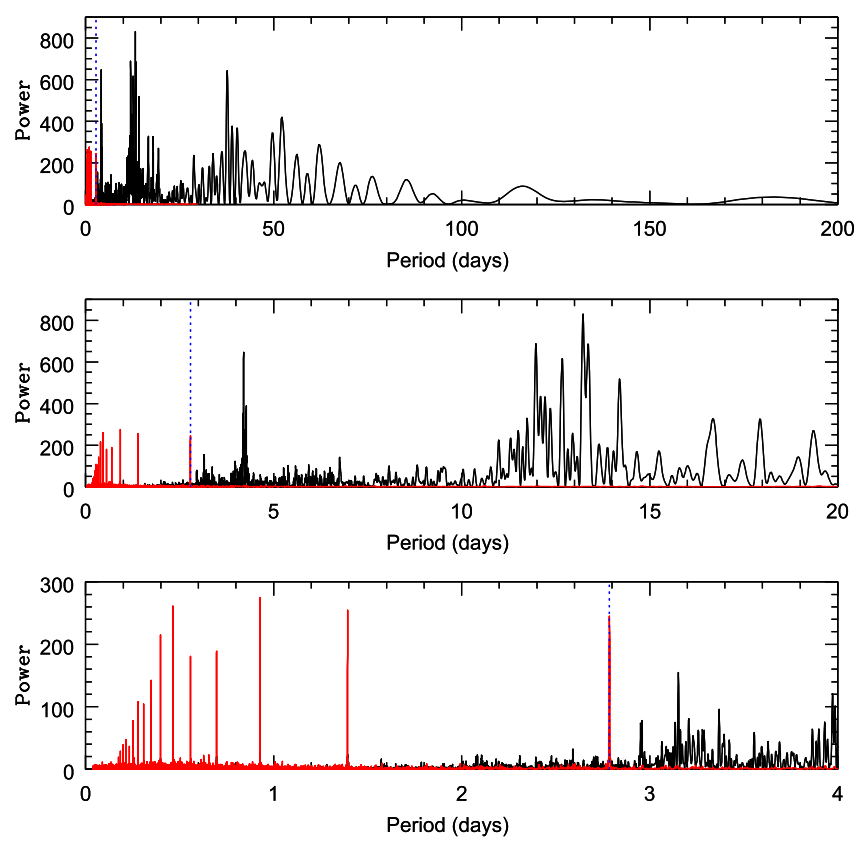

Figure 3. GLS periodograms of the DV light curve (red) and the PCA light curve (black). The vertical dashed, blue line indicates the period of Kepler-21b $(P=2.7858$ days $)$.

Although Kepler-21, at $V=8.25$, is moderately saturated on the detector, both detrendings include all the saturated pixels. Since charge is conserved on the Kepler CCDs to a very high degree, accurate differential photometry is achievable for saturated objects, as long as enough pixels capture all the saturated regions and a significant amount of the star's pointspread function (Koch et al. 2010).

\subsection{HARPS-N Spectroscopy}

We collected a total of 82 (RV) observations of Kepler-21 with the HARPS-N spectrograph installed on the $3.6 \mathrm{~m}$ Telescopio Nazionale Galileo (TNG) at the Observatorio del Roque de los Muchachos in La Palma, Spain (Cosentino et al. 2012). HARPS-N is an updated version of HARPS at the ESO $3.6 \mathrm{~m}$ (Mayor et al. 2003), and has already produced a series of high-precision RV results (e.g., Covino et al. 2013; Pepe et al. 2013; Bonomo et al. 2014; Desidera et al. 2014; Dumusque et al. 2014; Esposito et al. 2014; López-Morales et al. 2014; Damasso et al. 2015; Dressing et al. 2015; Mancini et al. 2015; Motalebi et al. 2015; Sozzetti et al. 2015; Buchhave et al. 2016; Gettel et al. 2016; Malavolta et al. 2016).

We observed Kepler-21 between 2014 April and 2015 June as part of the HARPS-N Collaboration's Guaranteed Time Observations (GTO) program, following a standard observing approach of one or two observations per night, separated by $2-3 \mathrm{hr}$, on nights assigned to the GTO program. Kepler- 21 is a bright target with $V=8.25\left(K_{\mathrm{p}}=8.2\right)$, so we obtained spectra with signal-to-noise ratios $(\mathrm{S} / \mathrm{N})$ in the range $\mathrm{S} / \mathrm{N}=45-308$ (average $\mathrm{S} / \mathrm{N}=167$ ), at $550 \mathrm{~nm}$ in 10-30 minute exposures, depending on the seeing and sky transparency. A summary of the observations is provided in Table 4.

The average RV error of the observations is $1.59 \pm 0.68$ $\mathrm{m} \mathrm{s}^{-1}$. This value is larger than the expected error of about $1.00 \mathrm{~m} \mathrm{~s}^{-1}$ for a slowly rotating $\mathrm{F}$ - or G-dwarf of similar 


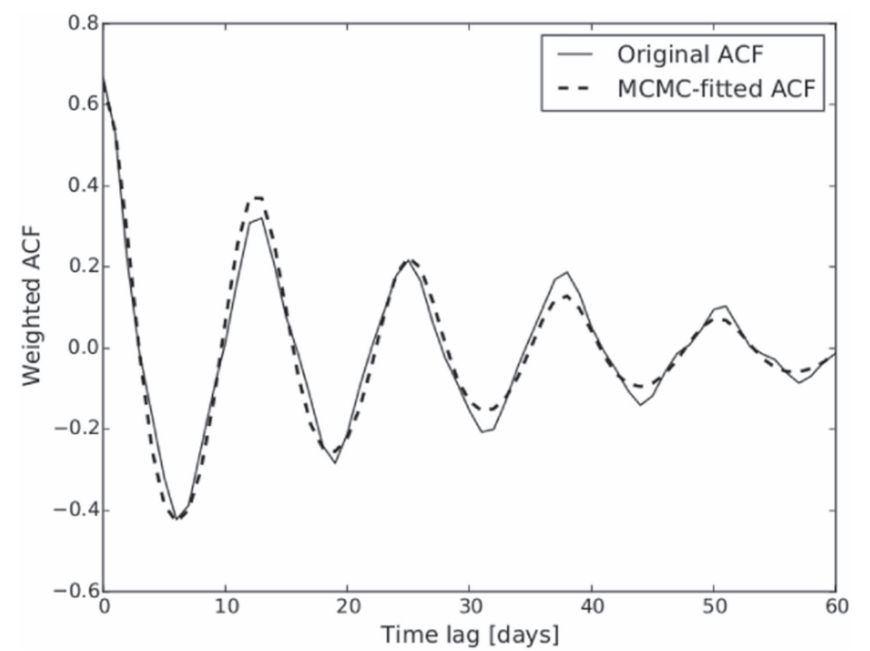

Figure 4. ACF of the Kepler-21 light curve (solid line), and the resulting Markov Chain Monte Carlo (MCMC) model of that function using a uSHO (dashed line).

apparent magnitude, but we attribute it to the faster rotation of this $\operatorname{star}\left(v \sin i_{\star}=8.4 \mathrm{~km} \mathrm{~s}^{-1}\right.$; see Section 3.3), which broadens the spectral lines and, therefore, gives a larger uncertainty on the RV determination. In addition, Kepler-21 presents significant photometric and spectroscopy variability, which produces an observed RV variation semiamplitude of about 10 $\mathrm{m} \mathrm{s}^{-1}$, including the stellar and planetary signals.

The spectra were reduced with version 3.7 of the HARPS-N Data Reduction Software (DRS), which includes corrections for color systematics introduced by variations in seeing (Cosentino et al. 2014). The radial velocities were computed using a numerical weighted mask following the methodology outlined by Baranne et al. (1996). The resultant radial velocities are presented in Table 4 and in Figure 2. Table 4 also includes each observation's central BJD, exposure time, bisector span and the measured $\log R_{\mathrm{HK}}^{\prime}$ activity index.

\subsection{HIRES Spectroscopy}

Howell et al. (2012) published 14 RV observations of Kepler-21 collected between 2010 August 31 and November 21 with HIRES on Keck. They adopted a different observing strategy than the one we used with HARPS-N: they observed the target in groups of three consecutive exposures, each lasting between 2 and 3 minutes in order to maintain a typical $\mathrm{S} / \mathrm{N}$ of about 210 and an internal RV error of approximately $2 \mathrm{~m} \mathrm{~s}^{-1}$ per exposure. The sampling of each group of exposures varied from twice a night to once every fifteen days. In total, they collected 13 groups of three exposures in this manner over a period of 51 days and a final single exposure 31 days later. The resultant RVs are reported in the original Howell et al. (2012) paper, and we also show them here together with the new HARPS-N RVs in Figure 2.

\section{ANALYSIS OF THE PHOTOMETRIC AND RV DATA}

\subsection{Preliminary Analysis of the Light Curves}

From the DV and the PCA light curves shown in Figure 1, the PCA light curve, which preserves the variability signal from the star, reveals stellar variability with a standard
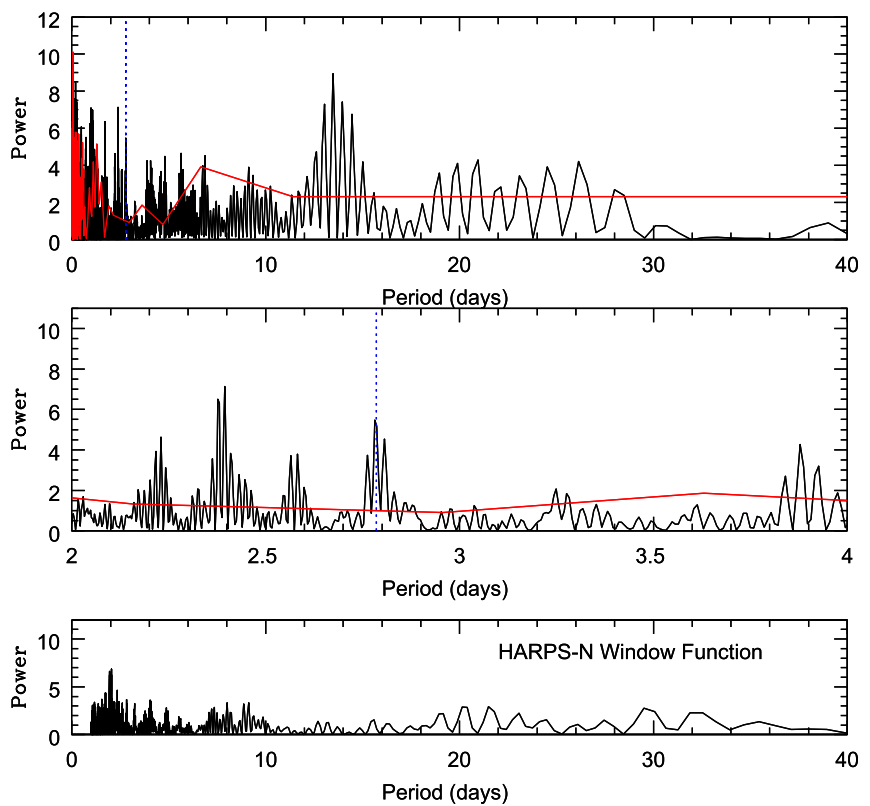

Figure 5. (Top, middle) GLS periodograms of the HIRES (red) and the HARPS-N (black) radial velocities. The vertical dashed, blue line indicates the period of Kepler-21b ( $P=2.7858$ days). The signal from the planet is visible in the HARPS-N RVs, but not in the HIRES RVs. (Bottom) Window function GLS periodogram of the HARPS-N RVs.

deviation of $145 \mathrm{ppm}$ and peak to peak variations of about $1300 \mathrm{ppm}$. In the DV light curve, after eliminating in-transit points, the standard deviation of the light-curve baseline gets reduced to $53 \mathrm{ppm}$. Figure 3 shows superimposed Generalized Lomb-Scargle (GLS, Zechmeister \& Kürster 2009) periodograms of the PCA and DV light curves. In the case of the PCA light curve, several strong peaks with $P \lesssim 50$ days dominate the periodogram. The strongest peak is at a period of 13.25 days, with several other strong peaks near that value. There are also strong, isolated peaks at 4.2 and 37.7 days, which are $1 / 3$ of, and three times the rotation period of the star found using autocorrelation functions (ACFs), as detailed below. The orbital period of Kepler-21b is not visible in the PCA light curve's periodogram. However, the periodogram of the DV light curve, where stellar variability has been removed and only the planetary transits remain, shows clearly a peak at a period of 2.7858 days, and its harmonics (e.g., 1.39 days, 0.92 days). There is no other significant peak in the DV light-curve periodogram.

To obtain a better estimation of the stellar rotation period and measure the star spots' decay times we applied an ACF to the PCA light curve. We produced the ACF by introducing discrete time lags, as described by Edelson \& Krolik (1988), in the light curve and cross-correlating the shifted light curves with the original, unshifted curve. The result is illustrated in Figure 4. The ACF peaks in the figure correspond to time offsets, which coincide with an integer multiple of the rotation period of the star. In addition, the effective decay time of the spots can be estimated by measuring the amplitude decay of the ACF side lobes in the figure. The amplitude decay occurs as the spots fade away with time. To measure these two parameters, we fitted the positive ACF lobes to the equation of motion for an underdamped simple harmonic oscillator (uSHO), which has a similar shape to the ACF. However, it has also been found that 

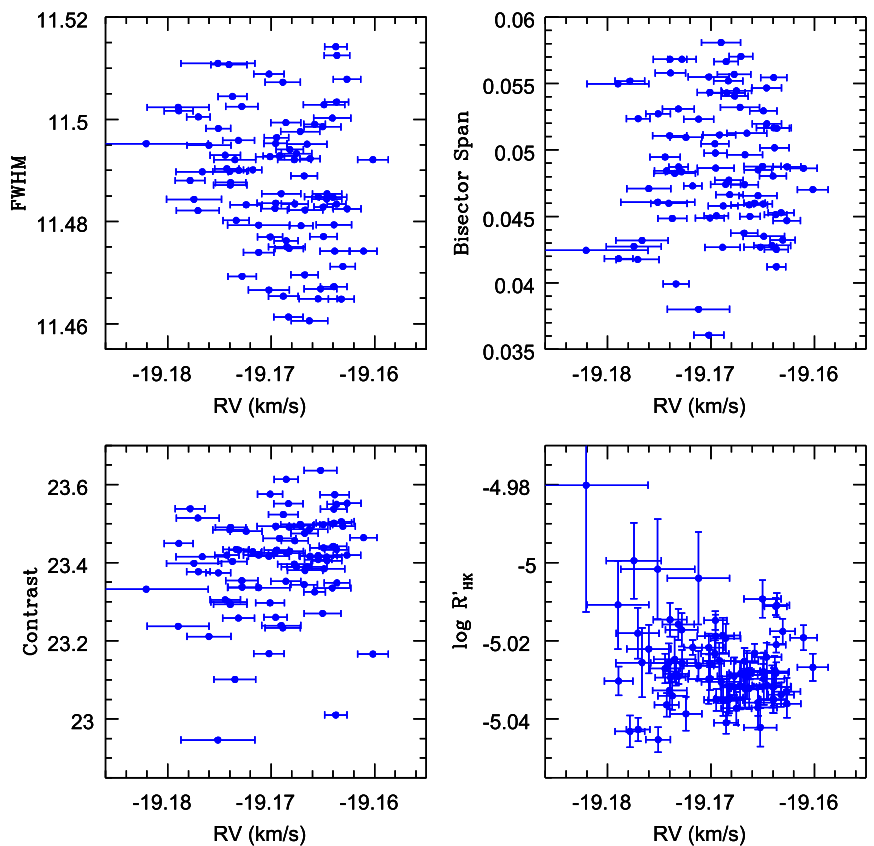

Figure 6. Plots of the $R_{\mathrm{HK}}^{\prime}$ activity index and the FWHM, bisector velocity span, and contrast of the CCF vs. the RV measurements from the HARPS-N data. There are no apparent correlations.

a large number of stars exhibit interpulses, which occur when an additional large spot appears on the opposite side of the star, introducing additional side lobes at half periods (H. A. C. Giles \& A. C. Cameron 2016, in preparation). This can be accounted for by introducing an additional cosine term in the uSHO equation. Therefore, the uHSO equation used here has the form

$$
y=e^{-A t}(B \cos \omega t+C \cos 2 \omega t)+y_{0},
$$

where $A$ is the spot decay timescale of the ACF, in days ${ }^{-1}$, and $\omega$ is the frequency, also in days ${ }^{-1} . B$ and $C$ are coefficients representing the amplitudes of the cosine terms and $y_{0}$ is an offset term from $y=0$.

We fit the uSHO equation to the ACF using an MCMC method, with starting parameters determined from the ACF, and step sizes drawn from a Gaussian distribution with parameter errors as the variance. The MCMC was performed twice: first to find the highest likelihood values; and second to explore that likelihood peak for the optimum set of values. The errors and the step size in the second MCMC were refined using the variance of the last 5000 steps in the first MCMC fit. Convergence was reached when the median of all previous likelihood values was greater than the current likelihood (Charbonneau et al. 2008; Knutson et al. 2008). Using this technique, we find a stellar rotation period of $12.62 \pm 0.03$ days and a spot decay time of $24.0 \pm 0.1$ days $^{-1}$. We note that this stellar rotational period is slightly shorter than the 13.25 day period found using a GLS periodogram. As shown in Figure 3, there is a set of strong peaks between 11 and 15 days in the GLS periodogram of the PCA curve. That set of peaks is consistent with a period of 12.6 days, and we attribute the difference between the GLS and the ACF results to the spot decay time, which is not accounted for in the GLS periodogram, and the long time baseline of the Kepler light curve, which likely includes
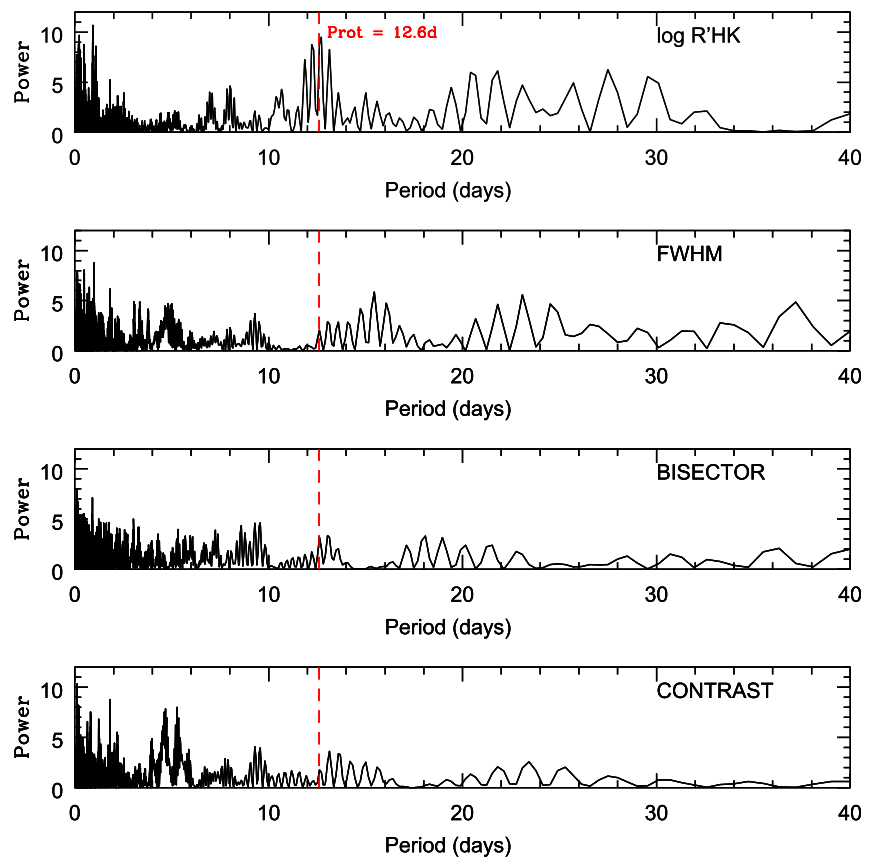

Figure 7. GLS periodograms of the $\log R_{\mathrm{HK}}^{\prime}$ activity index, FWHM, bisector span, and contrast values obtained from the HARPS-N spectra. The $R_{\mathrm{HK}}^{\prime}$ activity index periodogram shows a clear peak at 12.6 days.

many different, evolving spot configurations emerging at different rotation phases. We adopt the period of 12.6 days found by the ACF analysis as most reliable.

\subsection{Preliminary Analysis of the RV curves}

A GLS periodogram analysis of the HARPS-N radial velocities also reveals a complicated structure of peaks, as illustrated in Figure 5. In this case, the RV periodogram does show a peak at the period of Kepler-21b, but it is not the most significant peak. The strongest peak is at 13.47 days and there is a set of smaller peaks centered around that peak with similar structure to those in the PCA light-curve periodogram in Figure 3. We investigated whether that peak structure was produced by the observational window function, with negative result (see bottom panel of Figure 3 ). We conclude that this peak is the same as the one observed in the light curve at 13.25 days. Both peaks, and the pyramid-shaped structures of other strong peaks around them, result from the splitting of the rotational modulation peak arising from phase and amplitude changes as active regions grow and decay over the long time baseline of the observations.

Combining the 12.6 day period derived from the ACF analysis of the light curve with the $14.83 \pm 2.41$ day period derived in Section 3.3, we estimate an inclination for the spin axis for the star $i_{\star}=58_{-11}^{+32}$ degrees. With that result we cannot confirm a star-planet misalignment. The GLS periodogram of the HIRES RVs, shown in red in Figure 5, reveals no significant peak at the period of the planet, consistent with the nondetection reported by Howell et al. (2012).

\subsection{Stellar Parameters, Rotation and Activity}

Using the large number of high $\mathrm{S} / \mathrm{N}$, high-resolution spectra gathered by HARPS-N, we redetermined the stellar parameters 
Table 1

Median Values and 68\% Confidence Interval for Kepler-21b

\begin{tabular}{|c|c|c|}
\hline Parameter & Units & Value \\
\hline \multicolumn{3}{|l|}{ Stellar } \\
\hline$M$ & $\operatorname{Mass}\left(M_{\odot}\right)$ & $1.408_{-0.030}^{+0.021}$ \\
\hline$R$ & Radius $\left(R_{\odot}\right)$ & $1.902_{-0.012}^{+0.018}$ \\
\hline$L$ & Luminosity $\left(L_{\odot}\right)$ & $5.188_{-0.128}^{+0.142}$ \\
\hline$\rho_{*}$ & Density (cgs) & $0.287_{-0.005}^{+0.004}$ \\
\hline $\log \left(g_{*}\right)$ & Surface gravity (cgs) & $4.026 \pm 0.004$ \\
\hline$T_{\text {eff }}$ & $\begin{array}{l}\text { Effective temper- } \\
\text { ature }(\mathrm{K})\end{array}$ & $6305 \pm 50$ \\
\hline$[\mathrm{Fe} / \mathrm{H}]$ & Metallicity & $-0.03 \pm 0.10$ \\
\hline \multicolumn{3}{|l|}{ Planetary } \\
\hline$P$ & Period (days) & $2.7858212 \pm 0.0000032$ \\
\hline$a$ & Semimajor axis (AU) & $0.04285_{-0.00068}^{+0.00075}$ \\
\hline$R_{P}$ & Radius $\left(R_{\oplus}\right)$ & $1.639_{-0.015}^{+0.019}$ \\
\hline$T_{\text {eq }}$ & $\begin{array}{l}\text { Equilibrium temper- } \\
\text { ature }(\mathrm{K})\end{array}$ & $2025 \pm 20$ \\
\hline$\langle F\rangle$ & $\begin{array}{l}\text { Incident } \\
\quad \text { flux }\left(10^{9} \mathrm{erg} \mathrm{s}^{-1} \mathrm{~cm}^{-2}\right)\end{array}$ & $3.84 \pm 0.14$ \\
\hline \multicolumn{3}{|c|}{$\begin{array}{l}\text { Primary Transit } \\
\text { Parameters: }\end{array}$} \\
\hline$T_{C}$ & Time of transit $\left(\mathrm{BJD}_{\mathrm{TBD}}\right)$ & $2455093.83716_{-0.00085}^{+0.00082}$ \\
\hline$R_{P} / R$ & $\begin{array}{l}\text { Radius of planet in stellar } \\
\text { radii }\end{array}$ & $0.007885 \pm 0.000050$ \\
\hline$a / R$ & $\begin{array}{l}\text { Semimajor axis in stellar } \\
\text { radii }\end{array}$ & $4.929_{-0.047}^{+0.048}$ \\
\hline$u_{1}$ & $\begin{array}{l}\text { linear limb-darkening } \\
\text { coeff }\end{array}$ & $0.303_{-0.043}^{+0.044}$ \\
\hline$u_{2}$ & $\begin{array}{l}\text { quadratic limb-darkening } \\
\text { coeff }\end{array}$ & $0.296_{-0.046}^{+0.047}$ \\
\hline$i$ & Inclination (degrees) & $83.20_{-0.26}^{+0.28}$ \\
\hline$b$ & Impact parameter & $0.584_{-0.020}^{+0.018}$ \\
\hline$\delta$ & Transit depth & $0.00006217 \pm 0.00000078$ \\
\hline$T_{\text {FWHM }}$ & FWHM duration (days) & $0.1478_{-0.0018}^{+0.0019}$ \\
\hline$\tau$ & $\begin{array}{l}\text { Ingress/egress dura- } \\
\text { tion (days) }\end{array}$ & $0.001784_{-0.000050}^{+0.000051}$ \\
\hline$T_{14}$ & Total duration (days) & $0.1496 \pm 0.0019$ \\
\hline$P_{T}$ & $\begin{array}{l}\text { A priori nongrazing } \\
\text { transit probability }\end{array}$ & $0.2013_{-0.0019}^{+0.0020}$ \\
\hline$P_{T, G}$ & $\begin{array}{l}\text { A priori transit } \\
\text { probability }\end{array}$ & $0.2045 \pm 0.0020$ \\
\hline$F_{0}$ & Baseline flux & $1.00000223_{-0.00000016}^{+0.00000015}$ \\
\hline \multicolumn{3}{|c|}{$\begin{array}{l}\text { Secondary Eclipse } \\
\text { Parameters: }\end{array}$} \\
\hline$T_{S}$ & $\begin{array}{l}\text { Time of eclipse } \\
\left.\text { (BJD }_{\mathrm{TBD}}\right)\end{array}$ & $2455095.23007_{-0.00085}^{+0.00082}$ \\
\hline
\end{tabular}

Note.

${ }^{\text {a }}$ Values adopted from Silva Aguirre et al. (2015).

of Kepler-21 using the Stellar Parameter Classification pipeline (SPC; Buchhave et al. 2014), and the ARES+MOOG method described in Mortier et al. (2014). We analyzed 78 of the 82 spectra with exposure times larger than $900 \mathrm{~s}$ and a resolution of $R=115,000$ resulting in an average $\mathrm{S} / \mathrm{N}$ per resolution element of about 300 in the $\mathrm{MgB}$ line region. The remaining four spectra not included in the analysis had either lower $\mathrm{S} / \mathrm{N}$, because of shorter exposure times, or some artifact in the $\mathrm{MgB}$ line region. The stellar parameter values we obtain with the SPC are $T_{\text {eff }}=6216 \pm 50 \mathrm{~K}$ and $[\mathrm{Fe} / \mathrm{H}]=-0.06 \pm 0.08$,

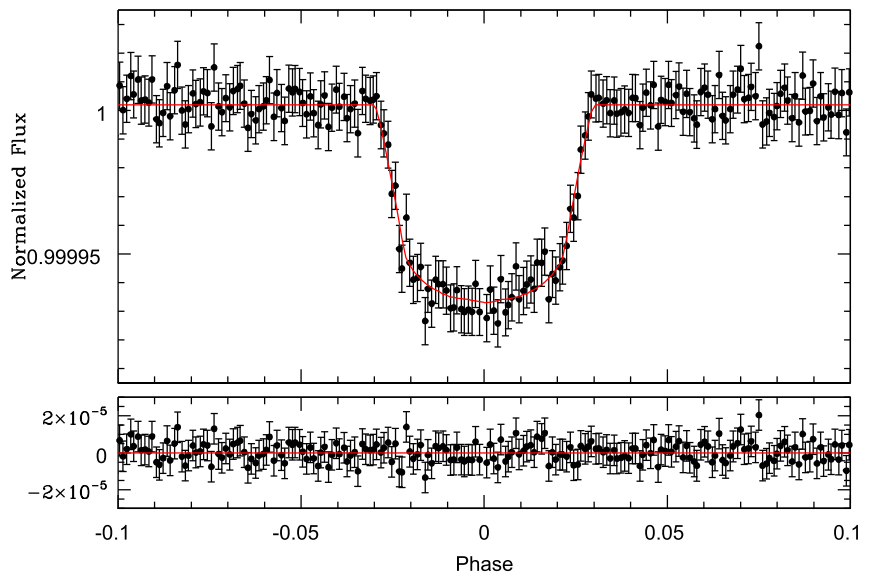

Figure 8. (Top) Normalized DV light curve, phased using the planet period reported in Table 1. The best-fit transit model is shown in red. The black dots show the light-curve data binned by a factor of 1000, including the corresponding error bars. (Bottom) Residuals to the transit fit.

when using the asteroseismic value of $\log (g)=4.019 \pm 0.009$ derived by Howell et al. (2012) as a prior. Adopting the more recent asteroseismic value of $\log (g)=4.026 \pm 0.004$ derived by Silva Aguirre et al. (2015) gives similar results. Leaving log $(g)$ as a free parameter in our fit yields $\log (g)=3.87 \pm 0.10$, slightly lower than the value reported by Howell et al. (2012) and Silva Aguirre et al. (2015), and a $T_{\text {eff }}=6127 \pm 49 \mathrm{~K}$ and $[\mathrm{Fe} / \mathrm{H}]=-0.11 \pm 0.08$. The parameter values obtained with the ARES+MOOG method are all consistent with the values from the SPC. We note that the $\log (g)$ derived from the HIRES spectra in Table 3 of Howell et al. (2012) also favors a lower value than the one yielded by the asteroseismology analysis. However, it has been previously shown that spectroscopic analyses are affected by degeneracies in $\log (g), T_{\text {eff }}$, and $[\mathrm{Fe} / \mathrm{H}]$, which generally result in an underestimation of $\log (g)$ (Torres et al. 2012). In addition, Kepler-21, being a bright star, has a Hipparcos parallax measurement of $8.86 \pm 0.58$ mas (van Leeuwen 2007), which corresponds to a distance for the system of $113 \pm 7 \mathrm{pc}$ and a stellar radius of $1.96 \pm 0.20 R_{\odot}$, in better agreement with the asteroseismology results.

Our analysis of the HARPS-N spectra yields a projected rotational velocity of $v \sin i_{\star}=8.4 \pm 0.5 \mathrm{~km} \mathrm{~s}^{-1}$. The error bars in the reported rotational velocity include uncertainties due to the line broadening by the spectrograph. From the HARPS$\mathrm{N}$ spectra, we also computed the $R_{\mathrm{HK}}^{\prime}$ activity index and several parameters of the cross-correlation function $(\mathrm{CCF})$, i.e., the FWHM, the bisector span, and the contrast, in the search for correlations with the RVs. We find no correlation between the RVs and any of those parameters, as illustrated in Figure 6. In addition, we derived the age and rotation period of the star following Mamajek \& Hillenbrand (2008). We estimate an age for the star of $3.03 \pm 0.35 \mathrm{Gyr}$, which agrees with the age of $2.84 \pm 0.35 \mathrm{Gyr}$ derived from asteroseismology (Howell et al. 2012), a rotational period of $P_{\mathrm{rot}}=14.83 \pm 2.41$ days, and a $\left\langle\log R_{\mathrm{HK}}^{\prime}\right\rangle=-5.027 \pm 0.011$.

As a note, we also performed a GLS periodogram analysis of the $R_{\mathrm{HK}}^{\prime}$ activity index values obtained from the HARPS-N spectra, as well as the FWHM, bisector span, and contrast values and find a clear peak in the periodogram of the $R_{\mathrm{HK}}^{\prime}$ index at 12.67 days (see Figure 7). This value coincides with the period of 12.6 days found by the ACF analysis of the 
Table 2

Best-fit Parameter Values of the RV Model for the HIRES-only, HARPS-N-only and Combined Data Sets

\begin{tabular}{|c|c|c|c|}
\hline & HIRES Only & HARPS-N Only & HIRES + HARPS-N \\
\hline \multicolumn{4}{|l|}{ Kepler-21b } \\
\hline$P[$ days $]$ & $2.78578(3)$ & $2.78574(3)$ & $2.78578(3)$ \\
\hline$t_{0, \mathrm{~b}}[\mathrm{BJD}-2450000]$ & $2456798.7188(1)$ & $2456798.7188(1)$ & 2456798.7188(1) \\
\hline$t_{\text {peri,b }}[$ BJD-2450000] & $2456798.2 \pm 0.7$ & $2456798.2 \pm 0.7$ & $2456797.9 \pm 0.7$ \\
\hline$K_{\mathrm{b}}\left[\mathrm{m} \mathrm{s}^{-1}\right]$ & $0.3 \pm 1$ & $2.12 \pm 0.66$ & $1.99 \pm 0.65$ \\
\hline$m_{\mathrm{b}}\left[M_{\oplus}\right]$ & $0.7 \pm 2.5$ & $5.41 \pm 1.76$ & $5.08 \pm 1.72$ \\
\hline$e_{\mathrm{b}}$ & $0.006 \pm 0.2$ & $0.007 \pm 0.1$ & $0.02 \pm 0.1$ \\
\hline$\omega_{\mathrm{b}}\left[^{\circ}\right]$ & $-106 \pm 104$ & $2 \pm 89$ & $-15 \pm 79$ \\
\hline$a_{\mathrm{b}}[\mathrm{AU}]$ & $0.0427172(3)$ & $0.0427172(3)$ & $0.0427172(3)$ \\
\hline \multicolumn{4}{|c|}{ Hyper-parameters of the GP } \\
\hline$\eta_{1}\left[\mathrm{~m} \mathrm{~s}^{-1}\right]$ & $8.9 \pm 6.6$ & $6.7 \pm 1.4$ & $8.6 \pm 1.4$ \\
\hline$\eta_{2}$ [days $]$ & $24.21 \pm 0.1$ & $24.04 \pm 0.09$ & $23.95 \pm 0.09$ \\
\hline$\eta_{3}$ [days $]$ & $12.61 \pm 0.02$ & $12.60 \pm 0.02$ & $12.63 \pm 0.02$ \\
\hline$\eta_{4}$ & $0.50 \pm 0.05$ & $0.42 \pm 0.05$ & $0.45 \pm 0.05$ \\
\hline \multicolumn{4}{|c|}{ Uncorrelated noise terms } \\
\hline$\sigma_{\text {hires }}\left[\mathrm{m} \mathrm{s}^{-1}\right]$ & $5.4 \pm 1.5$ & $\ldots$ & $4.9 \pm 1.4$ \\
\hline$\sigma_{\text {harpsn,instr }}\left[\mathrm{m} \mathrm{s}^{-1}\right]$ & $\cdots$ & $0.9 \pm 0.1$ & $1.0 \pm 0.1$ \\
\hline$\sigma_{\text {harpsn,gran }}\left[\mathrm{m} \mathrm{s}^{-1}\right]$ & $\cdots$ & $1.73 \pm 0.04$ & $1.50 \pm 0.03$ \\
\hline \multicolumn{4}{|l|}{ Systematic RV offsets } \\
\hline$R V_{0, \text { hires }}\left[\mathrm{m} \mathrm{s}^{-1}\right]$ & $-2.2 \pm 4.7$ & $\ldots$ & $-2.5 \pm 3.8$ \\
\hline$R V_{0, \text { harpsn }}\left[\mathrm{m} \mathrm{s}^{-1}\right]$ & $\ldots$ & $-10.0 \pm 1.6$ & $-10.5 \pm 2.0$ \\
\hline
\end{tabular}

Note. The numbers in parentheses represent the uncertainty in the last digit of the value.

Table 3

Parameters Modeled in the RV Analysis and their Prior Probability Distributions

\begin{tabular}{|c|c|c|}
\hline \multicolumn{3}{|c|}{ Kepler-21b orbital parameters } \\
\hline$P_{\text {orb }, \mathrm{b}}$ & Orbital period & Gaussian $\left(P_{\text {orb }, \mathrm{b}}, \sigma_{P_{\text {orb }, \mathrm{b}}}\right)$ \\
\hline$t_{0, \mathrm{~b}}$ & Transit ephemeris & $\operatorname{Gaussian}\left(t_{0, \mathrm{~b}}, \sigma_{\mathrm{t}_{0, \mathrm{~b}}}\right)$ \\
\hline$K_{b}$ & RV semiamplitude & $\begin{array}{l}\text { Modified Jeffreys } \\
\quad\left(\sigma_{\mathrm{RV}}, 2 \sigma_{\mathrm{RV}}\right)\end{array}$ \\
\hline$e_{\mathrm{b}}$ & Orbital eccentricity & Square root $[0,1]$ \\
\hline$\omega_{\mathrm{b}}$ & Argument of periastron & Uniform $[0,2 \pi]$ \\
\hline \multicolumn{3}{|c|}{ Hyper-parameters of the GP } \\
\hline$\eta_{1}$ & Amplitude of covariance & $\begin{array}{l}\text { Modified Jeffreys } \\
\quad\left(\sigma_{\mathrm{RV}}, 2 \sigma_{\mathrm{RV}}\right)\end{array}$ \\
\hline$\eta_{2}$ & Evolution timescale & $\operatorname{Gaussian}\left(T_{\mathrm{ev}}, \sigma_{T_{\mathrm{ev}}}\right)$ \\
\hline$\eta_{3}$ & Recurrence timescale & Gaussian $\left(P_{\text {rot }}, \sigma_{P_{\text {rot }}}\right)$ \\
\hline$\eta_{4}$ & Structure parameter & Gaussian $(0.5,0.05)$ \\
\hline \multicolumn{3}{|c|}{ Uncorrelated noise terms } \\
\hline$\sigma_{\text {hires }}$ & $\begin{array}{l}\text { HIRES instrument }+ \\
\text { granulation }\end{array}$ & Jeffreys $\left[0.01,10 \mathrm{~m} \mathrm{~s}^{-1}\right]$ \\
\hline$\sigma_{\text {harpsn,instr }}$ & HARPS-N instrument & Jeffreys $\left[0.01,10 \mathrm{~m} \mathrm{~s}^{-1}\right]$ \\
\hline$\sigma_{\text {harpsn,gran }}$ & HARPS-N granulation & Determined in MCMC \\
\hline \multicolumn{3}{|c|}{ Systematic RV offsets } \\
\hline$R V_{0, \text { hires }}$ & HIRES data set & Uniform \\
\hline$R V_{0, \text { harpsn }}$ & HARPS-N data set & Uniform \\
\hline
\end{tabular}

Note. For modified Jeffreys priors, the terms in the brackets refer to the knee and maximum value of the prior. In the case of a Gaussian prior, the terms within brackets represent the mean and standard deviation of the distribution. The terms within square brackets stand for the lower and upper limit of the specified distribution; if no interval is given, no limits are placed.
Kepler light curves in Section 3.1 and, therefore, reinforces the conclusion that 12.6 days corresponds to the rotation period of the star. The FWHM, bisector span, and contrast periodograms do not show any strong peaks around that period.

\section{LIGHT CURVE AND RV FITS}

\subsection{Kepler Light Curve}

We fit the transit of Kepler-21b using the detrended Kepler21b DV light curve shown in Section 2.1 and EXOFAST (Eastman et al. 2013). In the fit, which only uses the longcadence observations, we imposed Gaussian priors for the stellar parameters $T_{\text {eff }}=6305 \pm 50 \mathrm{~K},[\mathrm{Fe} / \mathrm{H}]=-0.03 \pm 0.10$, and $\log (g)=4.026 \pm 0.004$, based on the values reported by Silva Aguirre et al. (2015). We consider the parameters derived from asteroseismology more robust than those derived from the spectra, for the reasons explained in Section 3.3. We also introduced a Gaussian prior for the normalized light-curve baseline flux of $F_{0}=1.0000024 \pm 0.00000031$, to avoid systematic biases in the determination of the baseline flux introduced by in-transit points. We computed that normalized baseline flux beforehand by calculating the average and standard deviation of the light curve, not including the in-transit points and any other $3 \sigma$ outliers. The parameters fit are for the orbital period $P$, the transit epoch $T_{\mathrm{C}}$, the semimajor axis to stellar radius ratio $a / R_{*}$, the planet-to-star radius ratio $R p / R_{*}$, and the impact parameter $b=a / R_{*} \cos i$, where $i$ is the orbital inclination. We used a quadratic limb-darkening law, where the coefficients were not explicitly fit, but instead derived by interpolating the values in the Claret \& Bloemen (2011) tables for each value of $\log g, T_{\text {eff }}$, and $[\mathrm{Fe} / \mathrm{H}]$ in the fits. We modeled the system allowing for a nonzero eccentricity for Kepler-21b, 
Table 4

HARPS-N Radial Velocity Data ${ }^{a}$

\begin{tabular}{|c|c|c|c|c|c|c|}
\hline $\begin{array}{l}\text { BJD }_{\text {UTC }} \\
-2400000\end{array}$ & $\begin{array}{c}\mathrm{RV} \\
\left(\mathrm{m} \mathrm{s}^{-1}\right)\end{array}$ & $\begin{array}{c}\sigma_{\mathrm{RV}} \\
\left(\mathrm{m} \mathrm{s}^{-1}\right)\end{array}$ & $\begin{array}{l}\mathrm{BIS}_{\text {span }} \\
\left(\mathrm{m} \mathrm{s}^{-1}\right)\end{array}$ & $\begin{array}{c}\log R_{\mathrm{HK}}^{\prime} \\
(\mathrm{dex})\end{array}$ & $\begin{array}{c}\sigma \log R_{\mathrm{HK}}^{\prime} \\
\quad(\mathrm{dex})\end{array}$ & $\begin{array}{c}t_{\exp } \\
(\mathrm{s})\end{array}$ \\
\hline 56762.639368 & -19166.52 & 1.93 & 51.26 & -5.0287 & 0.0062 & 1800 \\
\hline 56764.647510 & -19168.57 & 1.45 & 47.41 & -5.0194 & 0.0038 & 1800 \\
\hline 56765.622661 & -19178.93 & 1.38 & 41.82 & -5.0303 & 0.0037 & 1200 \\
\hline 56766.637190 & -19182.07 & 5.99 & 42.44 & -4.9802 & 0.0324 & 900 \\
\hline 56768.669315 & -19174.07 & 1.75 & 45.98 & -5.0257 & 0.0050 & 1800 \\
\hline 56769.737457 & -19168.53 & 1.16 & 56.65 & -5.0410 & 0.0028 & 1200 \\
\hline 56783.561093 & -19175.10 & 1.19 & 52.72 & -5.0453 & 0.0032 & 1800 \\
\hline 56784.564843 & -19177.03 & 1.15 & 52.36 & -5.0427 & 0.0030 & 1800 \\
\hline 56785.617658 & -19177.83 & 1.43 & 55.19 & -5.0432 & 0.0041 & 1800 \\
\hline 56798.576307 & -19166.23 & 0.99 & 45.00 & -5.0277 & 0.0022 & 1800 \\
\hline 56799.561359 & -19168.19 & 1.32 & 46.64 & -5.0345 & 0.0034 & 900 \\
\hline 56800.551758 & -19167.10 & 1.14 & 57.04 & -5.0283 & 0.0029 & 1200 \\
\hline 56801.524205 & -19166.80 & 1.30 & 43.74 & -5.0288 & 0.0033 & 1200 \\
\hline 56802.539813 & -19164.89 & 2.00 & 43.50 & -5.0297 & 0.0067 & 1800 \\
\hline 56803.572851 & -19164.08 & 1.79 & 42.81 & -5.0317 & 0.0054 & 1800 \\
\hline 56813.516317 & -19171.77 & 0.88 & 47.29 & -5.0217 & 0.0019 & 1800 \\
\hline 56814.537057 & -19163.74 & 1.07 & 45.13 & -5.0112 & 0.0022 & 1800 \\
\hline 56816.639846 & -19163.67 & 0.90 & 41.21 & -5.0210 & 0.0020 & 1800 \\
\hline 56828.455942 & -19165.01 & 1.78 & 48.77 & -5.0093 & 0.0048 & 1800 \\
\hline 56829.423431 & -19163.63 & 1.23 & 51.63 & -5.0109 & 0.0031 & 1800 \\
\hline 56830.454195 & -19169.56 & 1.11 & 49.77 & -5.0148 & 0.0024 & 1800 \\
\hline 56831.713969 & -19165.78 & 1.04 & 46.00 & -5.0232 & 0.0024 & 1800 \\
\hline 56832.468881 & -19163.67 & 1.15 & 42.51 & -5.0282 & 0.0027 & 1800 \\
\hline 56833.531472 & -19173.73 & 1.38 & 44.85 & -5.0341 & 0.0036 & 1800 \\
\hline 56834.451861 & -19169.47 & 1.17 & 45.05 & -5.0351 & 0.0028 & 1800 \\
\hline 56835.497761 & -19174.28 & 1.18 & 48.43 & -5.0364 & 0.0030 & 1800 \\
\hline 56836.452270 & -19166.71 & 1.71 & 49.64 & -5.0323 & 0.0051 & 1800 \\
\hline 56836.614171 & -19164.59 & 1.42 & 51.97 & -5.0314 & 0.0037 & 1800 \\
\hline 56845.433549 & -19162.66 & 1.31 & 44.69 & -5.0330 & 0.0035 & 1800 \\
\hline 56846.481384 & -19173.12 & 0.94 & 48.73 & -5.0291 & 0.0020 & 1800 \\
\hline 56847.439704 & -19173.99 & 1.64 & 51.08 & -5.0327 & 0.0048 & 1800 \\
\hline 56848.402826 & -19167.54 & 1.46 & 54.46 & -5.0373 & 0.0042 & 1800 \\
\hline 56849.404363 & -19167.75 & 1.61 & 55.69 & -5.0291 & 0.0048 & 1800 \\
\hline 56850.412033 & -19171.20 & 3.00 & 38.01 & -5.0040 & 0.0118 & 1800 \\
\hline 56851.408417 & -19164.95 & 0.91 & 45.94 & -5.0280 & 0.0021 & 1800 \\
\hline 56852.407578 & -19172.83 & 1.56 & 48.37 & -5.0172 & 0.0043 & 1800 \\
\hline 56853.411021 & -19163.06 & 1.22 & 43.22 & -5.0176 & 0.0032 & 1800 \\
\hline 56863.638019 & -19177.08 & 2.06 & 41.76 & -5.0181 & 0.0065 & 1500 \\
\hline 56863.680716 & -19167.18 & 1.88 & 53.21 & -5.0315 & 0.0061 & 600 \\
\hline 56864.501604 & -19170.09 & 1.21 & 54.33 & -5.0297 & 0.0029 & 1500 \\
\hline 56864.622460 & -19164.62 & 1.43 & 54.67 & -5.0242 & 0.0037 & 900 \\
\hline 56865.515222 & -19167.71 & 1.30 & 54.05 & -5.0319 & 0.0032 & 900 \\
\hline 56865.651969 & -19163.92 & 1.69 & 51.67 & -5.0318 & 0.0048 & 600 \\
\hline 56866.489974 & -19172.41 & 1.54 & 50.95 & -5.0387 & 0.0043 & 900 \\
\hline 56866.602485 & -19176.65 & 2.53 & 43.19 & -5.0256 & 0.0089 & 600 \\
\hline 57115.684925 & -19170.21 & 1.97 & 55.50 & -5.0298 & 0.0063 & 900 \\
\hline 57122.653637 & -19173.91 & 1.47 & 55.80 & -5.0294 & 0.0042 & 900 \\
\hline 57156.575680 & -19175.15 & 3.58 & 46.06 & -5.0017 & 0.0128 & 900 \\
\hline 57158.600352 & -19177.45 & 2.66 & 42.74 & -4.9995 & 0.0097 & 900 \\
\hline 57159.594238 & -19169.55 & 1.78 & 48.63 & -5.0188 & 0.0055 & 900 \\
\hline 57160.591769 & -19170.20 & 1.46 & 36.07 & -5.0217 & 0.0035 & 900 \\
\hline 57160.701948 & -19173.38 & 1.25 & 39.92 & -5.0284 & 0.0031 & 900 \\
\hline 57161.578918 & -19173.95 & 1.62 & 56.82 & -5.0146 & 0.0043 & 900 \\
\hline 57161.695126 & -19172.80 & 1.37 & 56.83 & -5.0257 & 0.0034 & 900 \\
\hline 57180.506372 & -19164.00 & 1.32 & 48.04 & -5.0349 & 0.0035 & 900 \\
\hline 57180.722189 & -19169.61 & 1.35 & 50.47 & -5.0233 & 0.0034 & 900 \\
\hline 57181.488048 & -19163.85 & 1.41 & 50.17 & -5.0279 & 0.0040 & 900 \\
\hline 57181.727141 & -19161.05 & 1.30 & 48.62 & -5.0192 & 0.0032 & 900 \\
\hline 57182.478739 & -19169.18 & 1.58 & 51.14 & -5.0251 & 0.0046 & 900 \\
\hline 57182.689347 & -19164.93 & 1.32 & 52.94 & -5.0279 & 0.0033 & 900 \\
\hline
\end{tabular}


Table 4

(Continued)

\begin{tabular}{|c|c|c|c|c|c|c|}
\hline $\begin{array}{l}\text { BJD }_{\text {UTC }} \\
-2400000\end{array}$ & $\begin{array}{c}\mathrm{RV} \\
\left(\mathrm{m} \mathrm{s}^{-1}\right)\end{array}$ & $\begin{array}{c}\sigma_{\mathrm{RV}} \\
\left(\mathrm{m} \mathrm{s}^{-1}\right)\end{array}$ & $\begin{array}{l}\mathrm{BIS}_{\text {span }} \\
\left(\mathrm{m} \mathrm{s}^{-1}\right)\end{array}$ & $\begin{array}{c}\log R_{\mathrm{HK}}^{\prime} \\
(\mathrm{dex})\end{array}$ & $\begin{array}{c}\sigma \log R_{\mathrm{HK}}^{\prime} \\
(\mathrm{dex})\end{array}$ & $\begin{array}{r}t_{\exp } \\
(\mathrm{s})\end{array}$ \\
\hline 57183.511190 & -19173.15 & 1.55 & 53.09 & -5.0158 & 0.0040 & 900 \\
\hline 57183.717099 & -19168.87 & 1.71 & 42.67 & -5.0186 & 0.0045 & 900 \\
\hline 57184.479947 & -19178.99 & 3.00 & 54.98 & -5.0108 & 0.0113 & 900 \\
\hline 57184.690358 & -19176.01 & 2.12 & 47.09 & -5.0221 & 0.0062 & 900 \\
\hline 57185.477951 & -19174.57 & 1.47 & 49.48 & -5.0271 & 0.0038 & 900 \\
\hline 57185.681916 & -19171.39 & 1.45 & 52.06 & -5.0264 & 0.0038 & 900 \\
\hline 57186.475909 & -19168.82 & 1.42 & 45.79 & -5.0353 & 0.0039 & 900 \\
\hline 57186.704226 & -19163.21 & 1.25 & 45.28 & -5.0338 & 0.0031 & 900 \\
\hline 57188.483535 & -19168.47 & 1.78 & 54.30 & -5.0352 & 0.0054 & 900 \\
\hline 57188.698634 & -19168.32 & 1.42 & 55.20 & -5.0352 & 0.0039 & 900 \\
\hline 57189.475993 & -19173.50 & 2.02 & 48.24 & -5.0248 & 0.0059 & 900 \\
\hline 57189.690663 & -19169.02 & 1.90 & 58.09 & -5.0305 & 0.0055 & 900 \\
\hline 57190.488312 & -19165.22 & 1.57 & 42.69 & -5.0422 & 0.0049 & 900 \\
\hline 57190.704741 & -19162.65 & 1.34 & 48.74 & -5.0361 & 0.0036 & 900 \\
\hline 57191.488650 & -19163.93 & 1.28 & 55.45 & -5.0351 & 0.0034 & 900 \\
\hline 57191.704385 & -19166.76 & 1.33 & 47.40 & -5.0252 & 0.0034 & 900 \\
\hline 57192.485794 & -19168.26 & 1.37 & 47.73 & -5.0348 & 0.0037 & 900 \\
\hline 57192.702373 & -19170.10 & 1.39 & 44.88 & -5.0258 & 0.0035 & 900 \\
\hline 57193.488920 & -19160.15 & 1.47 & 47.02 & -5.0268 & 0.0035 & 900 \\
\hline 57193.703220 & -19165.44 & 1.32 & 48.50 & -5.0358 & 0.0034 & 900 \\
\hline 57195.488609 & -19165.45 & 1.84 & 46.56 & -5.0371 & 0.0056 & 900 \\
\hline 57195.637281 & -19166.29 & 1.75 & 45.87 & -5.0322 & 0.0053 & 900 \\
\hline
\end{tabular}

(This table is available in machine-readable form.)

but found solutions consistent with a circular orbit, and also consistent with the analysis of the RVs. The results of the final fit, assuming a circular orbit, are summarized in Table 1, which also includes a series of other parameters of the system computed by EXOFAST, e.g., the incident stellar flux in the surface of the planet, the transit probability, and the secondary eclipse time. The fit to the transit is also illustrated in Figure 8. The parameter uncertainties in the table are derived using a Differential Evolution Markov Chain Monte Carlo method, as described in detail in Section 2.2 of Eastman et al. (2013).

\subsection{Radial Velocities}

The RV, photometric, and $\log R_{\mathrm{HK}}^{\prime}$ activity index time series of Kepler-21 all show clear modulation around the stellar rotation period, as illustrated in Figures 3, 5, and 7, which suggest the star is moderately active. Stellar activity hinders the detectability of the planetary signals in RV curves, but recent studies (Haywood et al. 2014; Grunblatt et al. 2015; Rajpaul et al. 2015; Faria et al. 2016), have succeeded on modeling the activity and extracted the planetary Doppler signals using GP. In an effort to extract the RV signal of Kepler-21b from the available data, we model the orbit of Kepler-21b as a Keplerian with free eccentricity, and model the correlated noise introduced by rotation-modulated stellar activity using a GP with a quasi-periodic covariance Kernel of the form

$$
k\left(t, t^{\prime}\right)=\eta_{1}^{2} \cdot \exp \left[-\frac{\left(t-t^{\prime}\right)^{2}}{\eta_{2}^{2}}-\frac{\sin ^{2}\left(\frac{\pi\left(t-t^{\prime}\right)}{\eta_{3}}\right)}{\eta_{4}^{2}}\right],
$$

where the hyper-parameter $\eta_{1}$ is the amplitude of the covariance function, $\eta_{2}$ is equivalent to the evolution timescale of features in the stellar surface, which produce activity-induced RV variations, $\eta_{3}$ is equivalent to the stellar rotation period, and $\eta_{4}$ gives a measure of the level of high-frequency variability structure in the GP model. Our approach is similar to the ones used by Haywood et al. (2014), Grunblatt et al. (2015) and Faria et al. (2016), except that we set all the hyper-parameters as free parameters in the RV model. We leave $\eta_{1}$ as a free parameter, only constrained with a modified Jeffreys prior, as listed in Table 3. $\eta_{2}$ and $\eta_{3}$ are constrained with Gaussian priors using the values for the stellar rotation period and the active regions' lifetime (or spot decay time) determined via the ACF analysis described in Section 3.1. We constrain $\eta_{4}$ with a Gaussian prior centered around $0.5 \pm 0.05$. This value, which is adopted based on experience from previous data sets, allows the RV curve to have up to two or three maxima and minima, as is typical of stellar light curves and RV curves. Foreshortening of spots and other stellar surface features at the limb, and stellar limb-darkening smooth stellar photometric and RV variations, which means that a curve with more structure than that allowed by this value of $\eta_{4}$ would be unphysical. The strong constraints on the hyper-parameters, particularly $\eta_{4}$, are ultimately incorporated into the model likelihood and provide a realistic fit to the activity-induced variations, as shown in Figures 10 and 12. We note that GP is not only robust, but also extremely flexible. Our aim in this analysis is not to test how well an unconstrained GP can fit the data, but to use all the prior knowledge on the system to model the activity-driven signal as best as possible. 


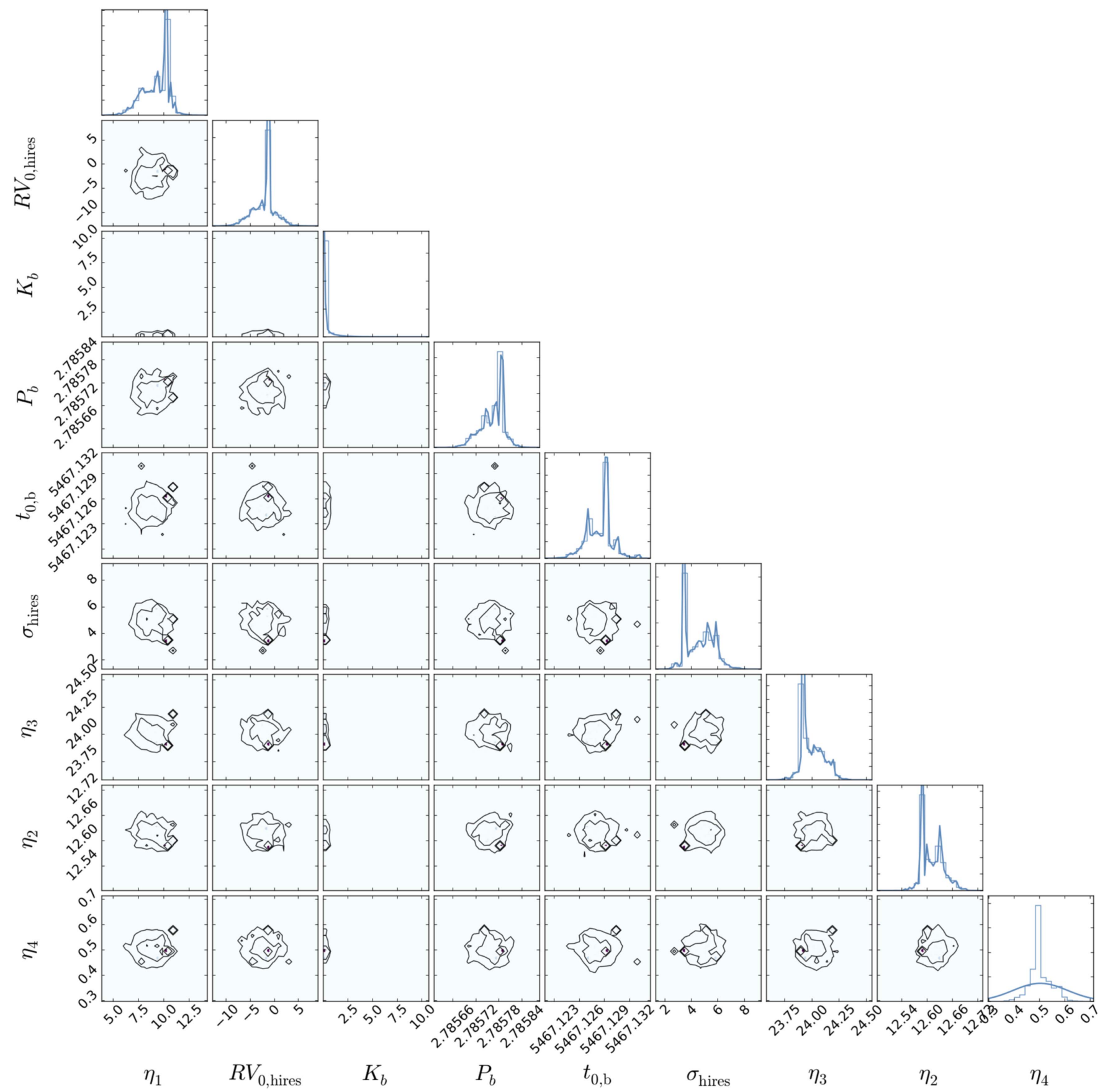

Figure 9. Marginalized 1D and 2D posterior distributions of the model parameters when fitting the HIRES campaign only. The solid lines overplotted on the histograms are kernel density estimations of the marginal distributions. It is clear that the MCMC chain has not converged, despite the additional priors imposed on $\eta_{1}$, $\mathrm{RV}_{0, \text { hires }}$, and $\sigma_{\text {hires }}$ (the eccentricity was also kept fixed to zero).

We introduce in the model jitter terms for each instrument data set. Kepler-21 is slightly evolved, thus its photosphere should have fewer and larger granules than main-sequence solar-type stars (Schwarzschild 1975; Antia et al. 1984; Mathur et al. 2011), and we expect larger activity signals in both the photometry and RVs during turnover convective timescales of a few hours. This is confirmed in both Figures 1 and 2 . Therefore, we expect uncorrelated noise in a timescale of only a few hours to be a combination of both instrument systematics and residual granulation and stellar oscillation motions. Regarding HARPS-N, recent observations of the Sun
(Dumusque et al. 2015, D. F. Phillips et al. 2016, in preparation) have shown that the instrument has a random day-to-day offset with an rms of $0.9 \mathrm{~m} \mathrm{~s}^{-1}$ (D. F. Phillips 2016, private communication). We account for this instrumental systematic by adding a noise term $\sigma_{\text {harpsn,instr }}=0.9 \pm 0.1$ $\mathrm{m} \mathrm{s}^{-1}$ in quadrature to the measured RV error bars. This value of $\sigma_{\text {harpsn, instr }}$ is constrained by a Gaussian prior. To estimate the granulation (and oscillation)-induced noise, we compute the inverse-variance weighted standard deviation of the residuals within each night, at each MCMC step, after subtracting the planet model. We note that this is only possible for the 2015 


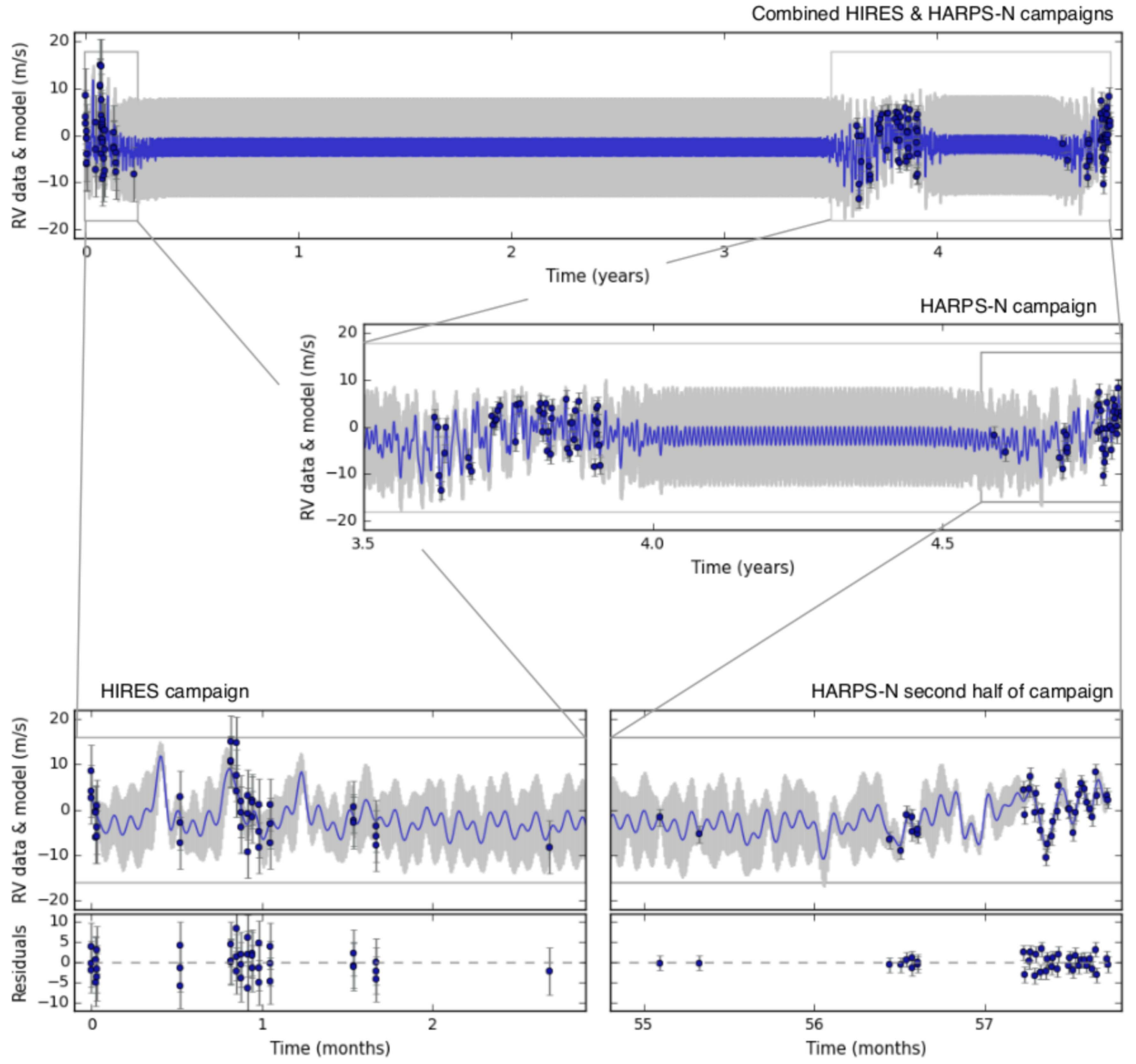

Figure 10. The HIRES and HARPS-N RV data (black points with error bars) and our best fit (blue line with gray shaded 1- $\sigma$ error regions), over various timescales: (top) the full combined data set spanning approximately 5 years - the first campaign was obtained with HIRES, while the second one is from HARPS-N; (middle) zoom-in on the HARPS-N data set spanning just under 1.5 years; (bottom left) zoom-in on the 3 month HIRES data set; (bottom right) zoom-in on a portion of HARPS-N data also covering 3 months. The two bottom panels, of equal timespan, show how the frequency structure of the model is preserved in time and adequately fits the observations throughout the combined data set. The residuals, after subtracting the model from the data, are shown for the two bottom panels (they are representative of the whole data set).

HARPS-N data set, which has two observations per night, separated by a few hours. This noise term, $\sigma_{\text {harpsn,gran, }}$, is then added in quadrature to the measured RV error bars, together with $\sigma_{\text {harpsn,instr. In the case of HIRES, they also collected }}$ several RV measurements per night, but those were collected consecutively, so they cannot be used to probe granulation over timescales of several hours. We also do not have additional information about the intrinsic instrumental systematics of HIRES, and so we use an overall free term, $\sigma_{\text {hires }}$, to account for both instrument and granulation noise.
We also adopt Gaussian priors for the orbital period of the planet and the phase of the transits in the Keplerian fit, using the best-fit values for those parameters computed in Section 4.1. Finally, we account for instrumental zero-point offsets of the two spectrographs with two separate terms, $\mathrm{RV}_{0 \text {,hires }}$, for HIRES and $\mathrm{RV}_{0 \text {,harpsn }}$ for HARPS-N. We summarize the priors used for each free parameter of our RV model in Table 3.

We fit the HIRES and HARPS-N data both separately and together. We maximize the likelihood of our model and determine the best-fit parameter values through an MCMC 


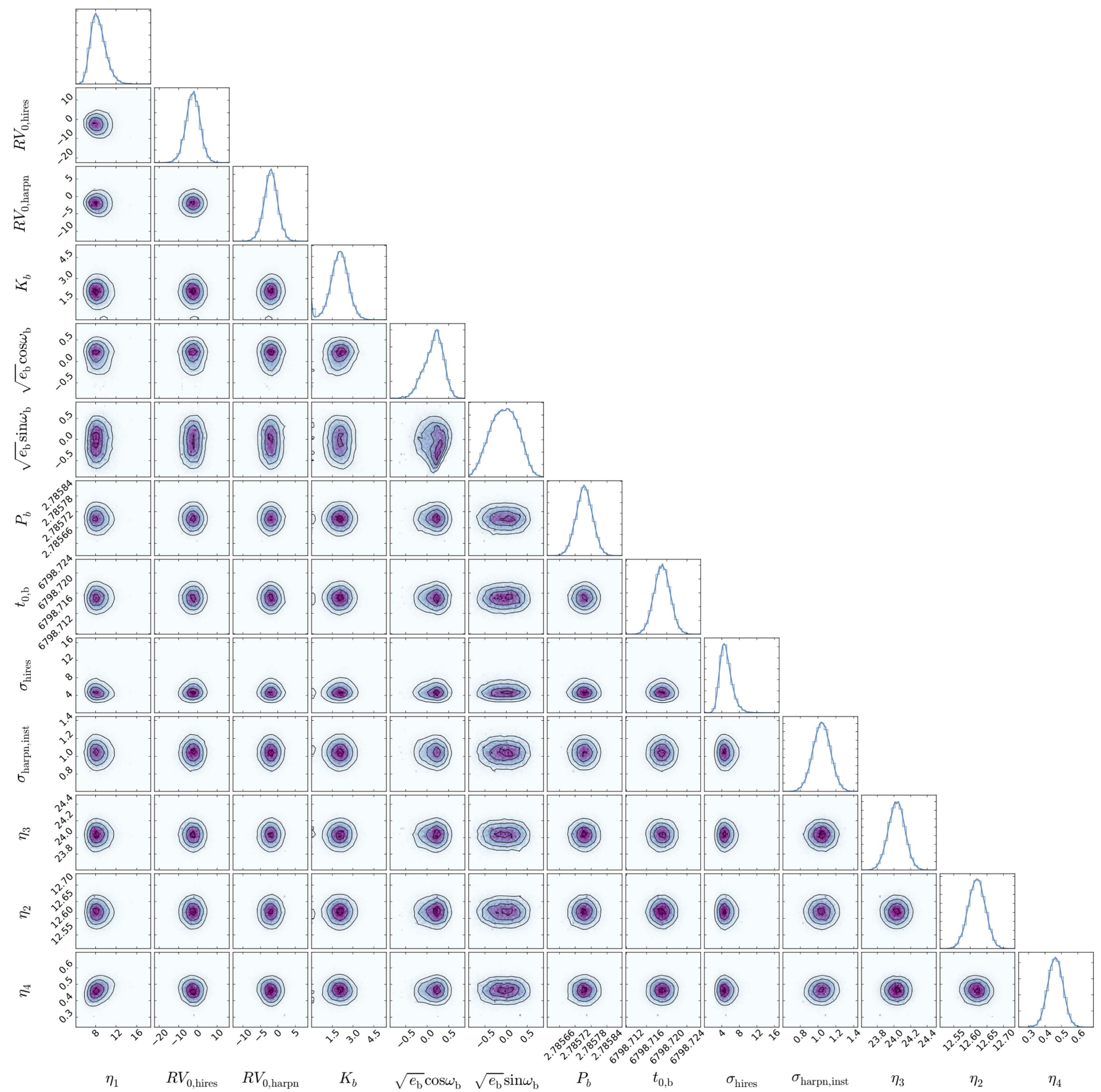

Figure 11. Marginalized 1D and 2D posterior distributions of the model parameters when fitting the HIRES and HARPS-N campaigns together. The solid lines overplotted on the histograms are kernel density estimations of the marginal distributions. These smooth, Gaussian-shaped posterior distributions attest to the good convergence of the MCMC chain.

procedure similar to the one described in Haywood et al. (2014). We ran the MCMC chains for 1,000,000 steps each, confirming their convergence using the Gelman-Rubin criterion (Gelman et al. 2004; Ford 2006). The best-fit parameters for all three runs are summarized in Table 2.

\subsubsection{HIRES-only Analysis}

The HIRES RV data set on its own yields no significant detection of Kepler-21b, as can be seen in column 1 of Table 2. Our MCMC chains did not converge within 1,000,000 steps. As a test to see whether the chain converged using a more tightly constrained model, we fixed the eccentricity to zero and imposed Gaussian priors on the parameters that had uninformative priors otherwise: $\eta_{1}, R V_{0 \text {, hires }}$ and $\sigma_{\text {hires }}$, but not $K_{\mathrm{b}}$. We centered these priors around the best-fit values of the combined HIRES and HARPS-N model. In spite of these constraints, the MCMC chains still do not converge, as illustrated in Figure 9. These findings are consistent with the nondetection result reported by Howell et al. (2012). We also note that our best-fit values for $\sigma_{\text {hires }}$ are consistent with the jitter value of $5 \mathrm{~m} \mathrm{~s}^{-1}$ reported by Howell et al. (2012).

We attribute the nondetection in the HIRES data to the adopted observing strategy. The HIRES data set consists of 40 observations over 80 days. The first 39 observations consisted of groups of three consecutive $150 \mathrm{~s}$ exposures, some collected 


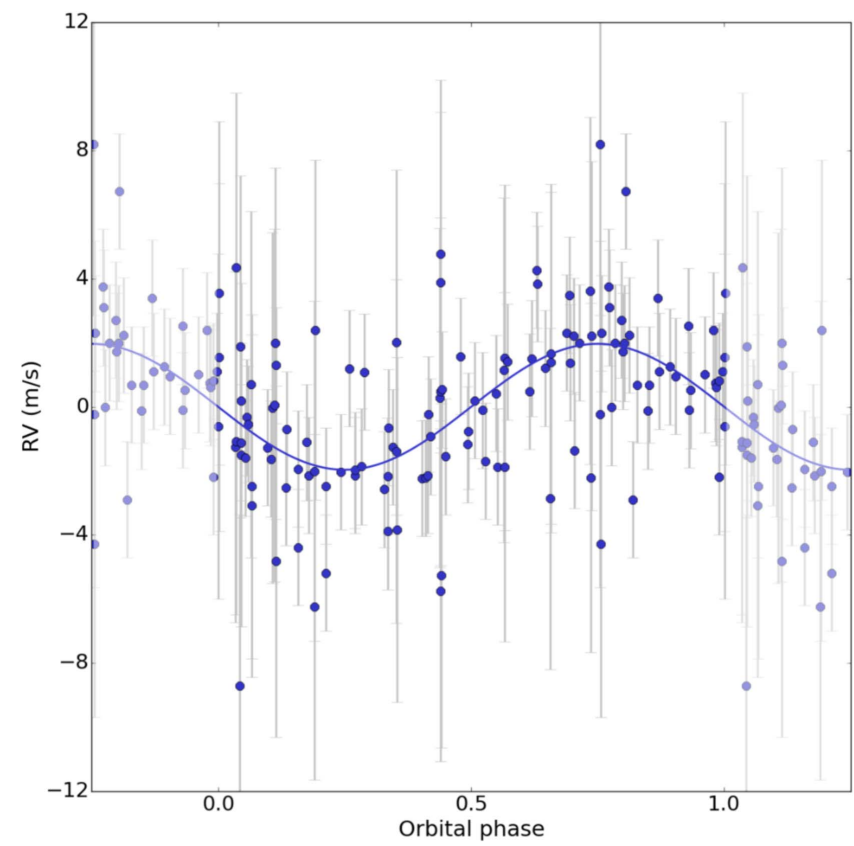

Figure 12. Phase plot of the orbit of Kepler-21b for the best-fit model after subtracting the GP component, for the combined HIRES and HARPS-N data sets.

on consecutive nights, and some with gaps of several nights between them. Each group of three consecutive observations was averaged out to produce an RV data point per night, equivalent to a 10 minute-long observation, and so the 40 observation data set is effectively equivalent to 14 observations. The largest stretch of consecutive nightly observations is five days, which is slightly less than two orbital periods of Kepler-21b. The full run spans 80 nights, which based on our analysis in Section 3.1, span three stellar active-region evolution timescales, and over six times the stellar rotation cycle. Therefore, the coherence in the activity-induced signal is lost and the sampling of the observations is too sparse to decouple the orbit of Kepler-21b from the activity-induced stellar variations.

\subsubsection{HARPS-N-only Analysis}

The HARPS-N data set, although its observing strategy was not fully tailored for this system either, does yield a detection of the Doppler signal of Kepler-21b and a mass measurement of $5.41 \pm 1.76 M_{\oplus}$ (see column 2 in Table 2). The HARPS-N observations are split into two seasons. During the 2014 season, of 45 observations spanning almost four active-region lifetimes and eight stellar rotation cycles, we collected a single observation per night, with the exception of one night in the middle of the run and the last four nights of the run, when we collected two observations per night. Two thirds of the observations were taken in three clusters of 6,7 , and 11 consecutive nights; the remaining third are scattered in groups of three nights or fewer. In the 2015 season, we deployed a different observing strategy, which proved to be better suited to characterizing the planet's orbit and the activity signal. In this season, we collected 37 observations: the first five were still one observation per night, on isolated nights; the other 32 observations were taken twice per night, separated always at least by 3.5 hours, but on average by five hours. The last 28 observations were taken on 15 consecutive nights, with gaps in two of the nights due to bad weather. This last stretch of observations fits well within one active-region lifetime (about two stellar rotation cycles), which means that the coherency of the activity-induced variations is preserved. However, we note that the 28 last observations alone do not yield a significant detection of Kepler-21b. The ideal number of observations, their sampling and stretch in time must be somewhere between these 28 observations and the full HARPS-N campaign, but exploring this further is beyond the scope of our analysis in this paper. However, we highlight that observational strategies customized for individual targets will yield significant improvement in our capacity to detect planetary Doppler signals in RV curves of active stars.

\subsubsection{HIRES and HARPS-N Combined Analysis}

Although the HIRES campaign alone does not contain sufficient information to provide a robust mass determination of Kepler-21b, those data are still compatible with the HARPS-N campaign, as illustrated in Figure 10, and their combined analysis yields system parameters fully consistent with the HARPS-N data alone, as shown in column 3 of Table 2. Figure 11 shows the posterior distributions of the MCMC analysis for the combined data sets. Looking closely at the correlation plots of $K_{\mathrm{b}}$ reveals, in addition to the main distribution peak, a local area of maximum likelihood near $K_{b}=0 \mathrm{~m} \mathrm{~s}^{-1}$, which is not present in the analysis of the HARPS-N data alone and is, therefore, introduced by the HIRES data. This may be interpreted as the HIRES data acting as a prior on the HARPS-N observations when we combine the two data sets. In this case, the influence of this peak is diminished by the larger sampling of the HARPS-N data and the posterior distribution of $K_{\mathrm{b}}$ is fully dominated by the HARPS-N data set (as shown in Table 2, this does not significantly affect the resultant planet mass). However, there may be cases in which the additional peak in the posterior distribution becomes more prominent, affecting the final fit. This could, for example, occur when combining data sets from different instruments with similar numbers of observations.

Given that the results from the analysis of the HARPS-N data alone and the combined data sets are consistent, we adopt the solution of the combined data sets (column 3 Table 2) as the best-fit parameter values for the system. The resulting best-fit model for the combined data set is shown in Figure 10, and the resultant phase folded orbit of Kepler-21b is shown in Figure 12 . We note that the value of $K_{\mathrm{b}}$ for each fit remains consistent within $1 \sigma$ regardless of our choice of covariance function, parameter distributions or initial parameter values, which attests to the robustness of this result. In addition, the best-fit parameters remain the same when we fix the orbital eccentricity to zero, which supports the result of a zero eccentricity measurement in Table 2.

\section{DISCUSSION AND CONCLUSIONS}

We measure a mass for Kepler-21b of $5.1 \pm 1.7 M_{\oplus}$ and derive a revised radius for the planet of $1.639+0.019 /-0.015$ $R_{\oplus}$, in agreement with the previous radius measurement of Howell et al. (2012). Those parameters combined yield a density for this object of $6.4 \pm 2.1 \mathrm{~g} \mathrm{~cm}^{-3}$, which suggests a 


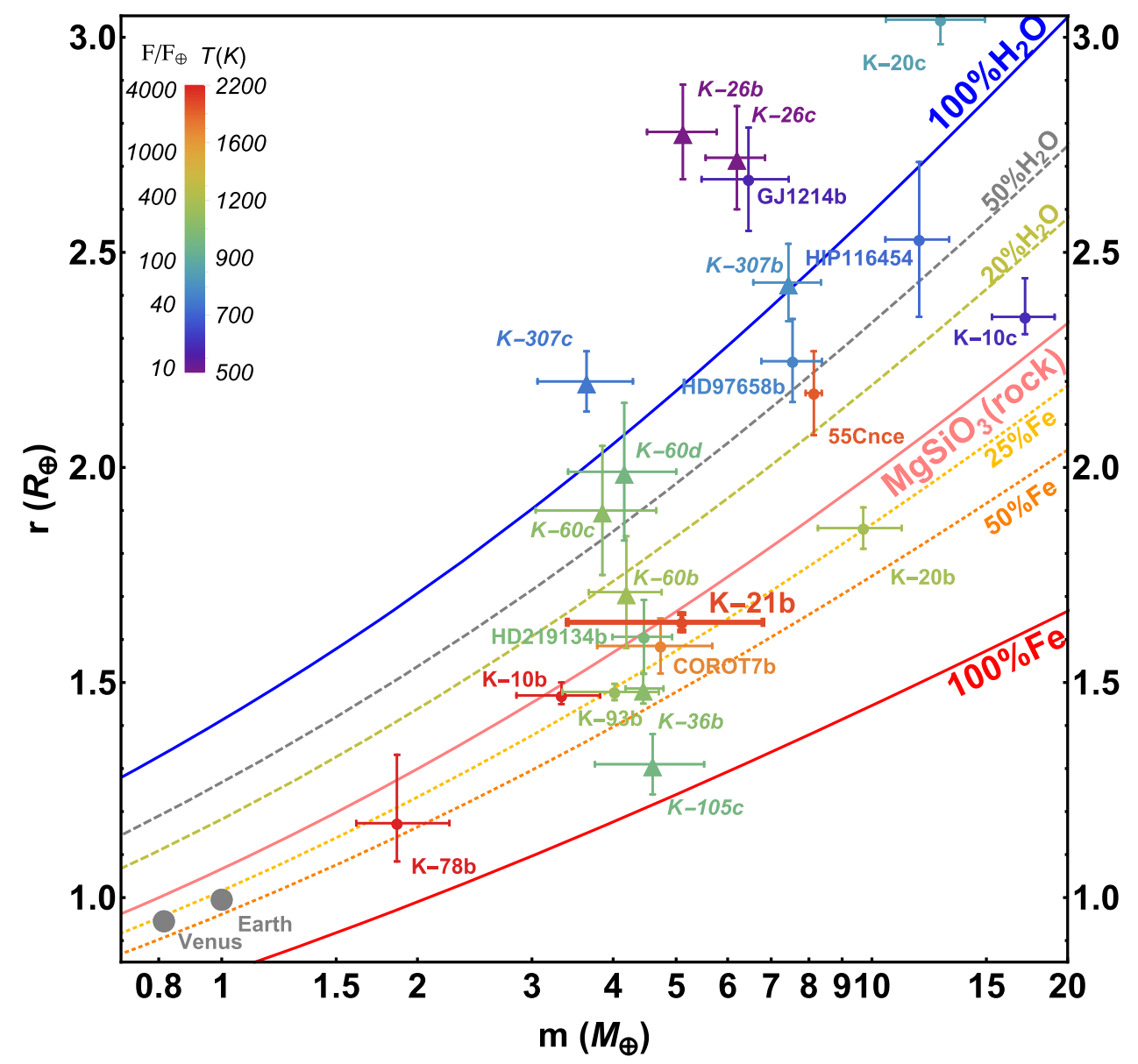

Figure 13. Mass-radius relation for planets with masses $<20 M_{\oplus}$, measured with precisions better than $20 \%$. Circles indicate the planets with masses measured via RVs; triangles indicate planets with masses measured via transit timing variations (Carter et al. 2012; Jontof-Hutter et al. 2016). The plot also includes Earth and Venus, for reference. The lines show models of different compositions, with solid lines indicating single composition planets (either $\mathrm{H}_{2} \mathrm{O}$, $\mathrm{MgSiO}_{3}$, i.e., rock, or Fe). The dashed and dotted lines indicate $\mathrm{Mg}$-silicate planets with different amounts of $\mathrm{H}_{2} \mathrm{O}$ and $\mathrm{Fe}$. The data points representing the planets are color coded as a function of incident bolometric stellar flux (compared to the Earth) and equilibrium temperature (assuming circular orbit, uniform planetary surface temperature, and bond albedo $A=0$ ). For other $A$ values, the temperature can be obtained by multiplying those values by a factor of $(1-A)^{1 / 4}$, following the flux and temperature scale indicated in the upper-left corner of the diagram.

rocky composition. Figure 13 shows theoretical mass-radius curves for planets composed of $100 \%, 50 \%$, and $25 \% \mathrm{H}_{2} \mathrm{O}$, as well as rocky planets with $25 \%, 50 \%$, and $100 \%$ Fe cores and the remaining mass in magnesium silicate mantles (Zeng et al. 2016). The figure also shows all the mass-radius measurements so far for exoplanets with masses less than $20 M_{\oplus}$ and mass errors smaller than $20 \%$. The location of Kepler- $21 \mathrm{~b}$ in this diagram is consistent with a rocky composition. Kepler-21b fits within the group of 1-6 $M_{\oplus}$ planets reported by Dressing et al. (2015) as being well described by the same fixed ratio of iron to magnesium silicate. The recently discovered Kepler-20b, with a mass of $9.7 M_{\oplus}$ also fits in that group (Buchhave et al. 2016). Kepler-21b also has similar parameters to CoRoT-7b (Barros et al. 2014; Haywood et al. 2014).

If the interior of Kepler-21b is differentiated, i.e., the Fe in the planet's interior has sunk to the center, while the lighter silicates remain in the mantle, we can use Equation (3) in Zeng et al. (2016) to estimate a core mass fraction (CMF) for this planet of $0.1 \pm 0.3$, which is, within the uncertainties, close to the CMF of 0.3 for Earth and Venus in the solar system. Most of the uncertainty in this CMF estimate comes from the current error in the mass and refining the mass measurement would yield a more accurate CMF estimate. Rocky planets of the same composition and the same mass, one differentiated, one undifferentiated, will have almost identical radius, within 1\%-2\% (Zeng \& Sasselov 2013), and so at present we cannot distinguish between these two scenarios given the current uncertainty in the radius of Kepler-21b of $1.2 \%$.

With an estimated equilibrium temperature of about $2000 \mathrm{~K}$, the top few-hundred-kilometer-thick layer of Kepler-21b is expected to be molten. However, the silicate (rocky) mantle underneath is expected to be solid due to the fact that the adiabat has a shallower slope than the melting curve (Stixrude 2014; Zeng \& Jacobsen 2016). The core of the planet is expected to be fully or partially molten due to its pressure density. An interior structure calculation for Kepler21b using the Manipulate Planet tool (Zeng \& Sasselov 2013, 2014; Zeng et al. 2016), gives a central pressure for the planet of around $1200 \mathrm{GPa}$. The pressure at the core-mantle boundary is estimated to be $800 \mathrm{GPa}$. The density at the planet's center is estimated to be about $17 \mathrm{~g} \mathrm{cc}^{-1}$, and so compared to the zeropressure density of iron $\left(7-8 \mathrm{~g} \mathrm{cc}^{-1}\right)$, there appears to be significant compression in the core. The density of silicate at the core-mantle boundary of the planet is estimated to be about $8 \mathrm{~g} \mathrm{cc}^{-1}$. 
As mentioned in the introduction, one possibility is that Kepler-21b is the remnant core of a hot gas giant, which has lost its envelope. In a recent paper by Jackson et al. (2016) the authors highlight Kepler-21b as one of the discovered short period planets, whose existence could be explained by the coupling of orbital evolution and roche-lobe overflow of a gas giant. Our mass measurement places the planet at the orbital separation predicted by this model.

Kepler-21b orbits the brightest planet host star discovered by the Kepler mission. The star is a slightly evolved F6IV subgiant, with intrinsic RV variations up to about $10 \mathrm{~m} \mathrm{~s}^{-1}$. With GP, however, we can reconstruct the intrinsic stellar variability well enough to confidently extract RV signals with amplitudes five times smaller than the stellar noise. The apparent brightness of Kepler-21 is similar to the bright targets to be observed by TESS and many of those targets will most likely have significant intrinsic RV variability. Therefore, this study serves as an example of the kind of RV analysis, which will be necessary to confirm the masses of TESS planet candidates. In particular, we emphasize the need for RV observations with cadence tailored for each target, based on their stellar rotational period and active-region lifetimes to efficiently model the activity and extract the planetary Doppler signal.

Given the proximity of Kepler-21b to its host star and with a planetary surface temperature of about $2000 \mathrm{~K}$, it is unlikely that the planet has retained a significant amount of envelope volatiles. However, even though the atmosphere of the planet is expected to be tenuous, the brightness of the system may allow detection of atmospheric features in the UV, optical or infrared, either from space, with $H S T$ and JWST, or from the ground with upcoming large facilities.

We thank the anonymous referee for helpful comments to the manuscript. The HARPS-N project has been funded by the Prodex Program of the Swiss Space Office (SSO), the Harvard University Origins of Life Initiative (HUOLI), the Scottish Universities Physics Alliance (SUPA), the University of Geneva, the Smithsonian Astrophysical Observatory (SAO), and the Italian National Astrophysical Institute (INAF), the University of St. Andrews, Queens University Belfast, and the University of Edinburgh. This publication was made possible through the support of a grant from the John Templeton Foundation. The opinions expressed are those of the authors and do not necessarily reflect the views of the John Templeton Foundation. This material is based upon work supported by the National Aeronautics and Space Administration under grant No. NNX15AC90G issued through the Exoplanets Research Program. The research leading to these results has received funding from the European Union Seventh Framework Programme (FP7/2007-2013) under grant Agreement No. 313014 (ETAEARTH). This work was performed in part under contract with the California Institute of Technology (Caltech)/Jet Propulsion Laboratory (JPL) funded by NASA through the Sagan Fellowship Program executed by the NASA Exoplanet Science Institute. P.F. acknowledges support by Fundação para a Cîencias e a Tecnologia (FCT) through Investigador FCT contract of reference IF/01037/2013 and POPH/FSE (EC) by FEDER funding through the program "Programa Operacional de Factores de Competitividade COMPETE", and further support in the form of an exploratory project of reference IF/01037/2013CP1191/CT0001. L.Z. is supported by a grant from the Simons Foundation (SCOL, award \#337090). A.V. is supported by the NSF Graduate Research Fellowship, Grant No. DGE 1144152. X.D. is grateful to the Society in Science-Branco Weiss Fellowship for its financial support.

\section{Facilities: TNG: HARPS-N, Keck: HIRES, TRES.}

\section{REFERENCES}

Antia, H. M., Chitre, S. M., \& Narasimha, D. 1984, ApJ, 282, 574 Baranne, A., Queloz, D., Mayor, M., et al. 1996, A\&AS, 119, 373 Barros, S. C. C., Almenara, J. M., Deleuil, M., et al. 2014, A\&A, 569, A74 Bonomo, A. S., Sozzetti, A., Lovis, C., et al. 2014, A\&A, 572, A2 Buchhave, L. A., Bizzarro, M., Latham, D. W., et al. 2014, Natur, 509, 593 Buchhave, L. A., Dressing, C., Dumusque, X., et al. 2016, AJ, 152, 160 Carter, J. A., Agol, E., Chaplin, W. J., et al. 2012, Sci, 337, 556 Charbonneau, D., Knutson, H. A., Barman, T., et al. 2008, ApJ, 686, 1341 Christiansen, J. L., Clarke, B. D., Burke, C. J., et al. 2013, ApJS, 207, 35 Claret, A., \& Bloemen, S. 2011, A\&A, 529, A75

Cosentino, R., Lovis, C., Pepe, F., et al. 2012, Proc. SPIE, 8446, 84461V Cosentino, R., Lovis, C., Pepe, F., et al. 2014, Proc. SPIE, 9147, 91478C Coughlin, J. L., \& López-Morales, M. 2012, AJ, 143, 39

Covino, E., Esposito, M., Barbieri, M., et al. 2013, A\&A, 554, A28

Damasso, M., Esposito, M., Nascimbeni, V., et al. 2015, A\&A, 581, L6 Desidera, S., Bonomo, A. S., Claudi, R. U., et al. 2014, A\&A, 567, L6

Dressing, C. D., \& Charbonneau, D. 2013, ApJ, 767, 95

Dressing, C. D., \& Charbonneau, D. 2015, ApJ, 807, 45

Dressing, C. D., Charbonneau, D., Dumusque, X., et al. 2015, ApJ, 800, 135 Dumusque, X., Bonomo, A. S., Haywood, R. D., et al. 2014, ApJ, 789, 154 Dumusque, X., Glenday, A., Phillips, D. F., et al. 2015, ApJL, 814, L21 Eastman, J., Gaudi, B. S., \& Agol, E. 2013, PASP, 125, 83

Edelson, R. A., \& Krolik, J. H. 1988, ApJ, 333, 646

Esposito, M., Covino, E., Mancini, L., et al. 2014, A\&A, 564, L13 Faria, J. P., Haywood, R. D., Brewer, B. J., et al. 2016, A\&A, 588, A31 Ford, E. B. 2006, ApJ, 642, 505

Foreman-Mackey, D., Hogg, D. W., \& Morton, T. D. 2014, ApJ, 795, 64 Fressin, F., Torres, G., Charbonneau, D., et al. 2013, ApJ, 766, 81

Gelman, A., Carlin, J. B., Stern, H. S., \& Rubin, D. B. 2004, Bayesian Data Analysis (London, Boca Raton, FL: Chapman and Hall, CRC Press)

Gettel, S., Charbonneau, D., Dressing, C. D., et al. 2016, ApJ, 816, 95 Grunblatt, S. K., Howard, A. W., \& Haywood, R. D. 2015, ApJ, 808, 127 Haywood, R. D., Collier Cameron, A., Queloz, D., et al. 2014, MNRAS, 443,2517

Howard, A. W., Marcy, G. W., Bryson, S. T., et al. 2012, ApJS, 201, 15

Howell, S. B., Rowe, J. F., Bryson, S. T., et al. 2012, ApJ, 746, 123 Inamdar, N. K., \& Schlichting, H. E. 2015, MNRAS, 448, 1751

Jenkins, J. M., Caldwell, D. A., Chandrasekaran, H., et al. 2010, ApJL, 713, L87

Jontof-Hutter, D., Ford, E. B., Rowe, J. F., et al. 2016, ApJ, 820, 39

Knutson, H. A., Charbonneau, D., Allen, L. E., Burrows, A., \& Megeath, S. T. 2008, ApJ, 673, 526

Koch, D. G., Borucki, W. J., Basri, G., et al. 2010, ApJL, 713, L79

Lopez, E. D., \& Fortney, J. J. 2013, ApJ, 776, 2

Lopez, E. D., Fortney, J. J., \& Miller, N. 2012, ApJ, 761, 59

López-Morales, M., Triaud, A. H. M. J., Rodler, F., et al. 2014, ApJL, 792, L31

Malavolta, L., Nascimbeni, V., Piotto, G., et al. 2016, A\&A, 588, A118

Mamajek, E. E., \& Hillenbrand, L. A. 2008, ApJ, 687, 1264

Mancini, L., Esposito, M., Covino, E., et al. 2015, A\&A, 579, A136

Mathur, S., Hekker, S., Trampedach, R., et al. 2011, ApJ, 741, 119

Mayor, M., Pepe, F., Queloz, D., et al. 2003, Msngr, 114, 20

McQuillan, A., Mazeh, T., \& Aigrain, S. 2014, ApJS, 211, 24

Mortier, A., Sousa, S. G., Adibekyan, V. Z., Brandão, I. M., \& Santos, N. C. 2014, A\&A, 572, A95

Motalebi, F., Udry, S., Gillon, M., et al. 2015, A\&A, 584, A72

Murtagh, F., \& Heck, A. 1987, A\&AS, 68, 113

Owen, J. E., \& Jackson, A. P. 2012, MNRAS, 425, 2931

Owen, J. E., \& Wu, Y. 2013, ApJ, 775, 105

Pepe, F., Cameron, A. C., Latham, D. W., et al. 2013, Natur, 503, 377

Petigura, E. A., Howard, A. W., \& Marcy, G. W. 2013a, PNAS, 110, 19273

Petigura, E. A., Marcy, G. W., \& Howard, A. W. 2013b, ApJ, 770, 69

Rajpaul, V., Aigrain, S., Osborne, M. A., Reece, S., \& Roberts, S. 2015 MNRAS, 452, 2269

Rasmussen, C. E., \& Williams, C. K. I. 2006, Gaussian Processes for Machine Learning (Cambridge, MA: MIT Press) 
Rogers, L. A. 2015, ApJ, 801, 41

Schlichting, H. E., Sari, R., \& Yalinewich, A. 2015, Icar, 247, 81

Schwarzschild, M. 1975, ApJ, 195, 137

Silburt, A., Gaidos, E., \& Wu, Y. 2015, ApJ, 799, 180

Silva Aguirre, V., Davies, G. R., Basu, S., et al. 2015, MNRAS, 452, 2127

Sozzetti, A., Bonomo, A. S., Biazzo, K., et al. 2015, A\&A, 575, L15

Stixrude, L. 2014, RSPTA, 372, 20130076

Torres, G., Fischer, D. A., Sozzetti, A., et al. 2012, ApJ, 757, 161 van Leeuwen, F. 2007, A\&A, 474, 653

Wu, H., Twicken, J. D., Tenenbaum, P., et al. 2010, Proc. SPIE, 7740, 774019

Zechmeister, M., \& Kürster, M. 2009, A\&A, 496, 577

Zeng, L., \& Jacobsen, S. B. 2016, ApJ, 829, 18

Zeng, L., \& Sasselov, D. 2014, ApJ, 784, 96

Zeng, L., \& Sasselov, D. D. 2013, PASP, 125, 2013

Zeng, L., Sasselov, D. D., \& Jacobsen, S. B. 2016, ApJ, 819, 127 